\title{
The isolated interacting galaxy pair NGC 5426/27 (Arp 271) ${ }^{\star}$
}

\author{
I. Fuentes-Carrera ${ }^{1}$, M. Rosado ${ }^{1}$, P. Amram ${ }^{2}$, D. Dultzin-Hacyan ${ }^{1}$, I. Cruz-González ${ }^{1}$, H. Salo ${ }^{3}$, E. Laurikainen ${ }^{3}$, \\ A. Bernal ${ }^{1}$, P. Ambrocio-Cruz ${ }^{1}$, and E. Le Coarer ${ }^{4}$ \\ ${ }^{1}$ Instituto de Astronomía, Universidad Nacional Autónoma de México (UNAM), Apdo. Postal 70-264, 04510, México, D.F., \\ México \\ 2 Laboratoire d'Astrophysique de Marseille, 2 place Le Verrier, Marseille Cedex 4, France \\ 3 Department of Physical Sciences, Division of Astronomy, University of Oulu, 90570 Oulu, Finland \\ ${ }^{4}$ Observatoire de Grenoble, BP 53X, 38041 Grenoble Cedex 9, France
}

Received 13 August 2003 / Accepted 30 September 2003

\begin{abstract}
We present $\mathrm{H} \alpha$ observations of the isolated interacting galaxy pair NGC 5426/27 using the scanning Fabry-Perot interferometer PUMA. The velocity field, various kinematical parameters and rotation curve for each galaxy were derived. The FWHM map and the residual velocities map were also computed to study the role of non-circular motions of the gas. Most of these motions can be associated with the presence of spiral arms and structure such as central bars. We found a small bar-like structure in NGC 5426, a distorted velocity field for NGC 5427 and a bridge-like feature between both galaxies which seems to be associated with NGC 5426. Using the observed rotation curves, a range of possible masses was computed for each galaxy. These were compared with the orbital mass of the pair derived from the relative motion of the participants. The rotation curve of each galaxy was also used to fit different mass distribution models considering the most common theoretical dark halo models. An analysis of the interaction process is presented and a possible 3D scenario for this encounter is also suggested.
\end{abstract}

Key words. galaxies: interactions - galaxies: kinematics and dynamics - galaxies: spiral - galaxies: individual: NGC 5426 galaxies: individual: NGC 5427

\section{Introduction}

Galaxies suffer modifications from close companions from the early stages of their lives. Encounters between galaxies are common and important phenomena in the evolution and transformation of these systems. The way the interacting process between two galaxies triggers, sustains or inhibits the formation of structure in spiral galaxies has been studied from different points of view, from broad statistical studies to more detailed morphological, photometric and kinematical analysis. Several attempts have been made to kinematically constraint the evolutionary scenario of interactions and the formation of structure such as bars, tidal arms, amongst others (Salo \& Laurikainen 1993, 2000a,b; Miwa \& Noguchi 1998; Kaufman et al. 1999). It has been found that although morphology is relatively easy to reproduce with numerical simulations (Toomre \& Toomre 1972; Barnes 1988; Barnes \& Hernquist 1996), kinematical information imposes important restrictions on the models applied (Salo \& Laurikainen 1993, 2000a,b). In these works, $\mathrm{N}$-body simulations have been compared with observations.

Send offprint requests to: I. Fuentes-Carrera,

e-mail: iqui@astroscu.unam.mx

$\star$ Table 1 is only available in electronic form at http://www. edpsciences.org
The predicted shape of the rotation curve, deviations of the gaseous spiral arms from the plane of the galactic disk, peculiar high velocities in certain directions of the galaxies and non-circular motions are constrained by $2 \mathrm{D}$ velocity fields produced by Fabry-Perot observations.

From the observational point of view, most of the work on the kinematics and dynamics of interacting galaxy pairs has been done using long-slit spectroscopy along certain predetermined positions thus restraining the kinematical information to a few points on the galaxy. Nevertheless, for a perturbed system, it is important to have kinematical information of larger portions of the disk using observational techniques such as extended-object Fabry-Perot scanning interferometry since extended kinematical information can help determine the effect the interacting process has had on each of the members of the interacting system and because axial symmetry is probably lost during the interaction (Marcelin et al. 1987; Amram et al. 1994, 2002).

The study of the dynamics of binary systems also allows one to estimate the total mass of a galaxy as well as its massto-luminosity ratio $(M / L)$. Through this method, the integrated mass of a component can be measured out to 10 times the luminous radius of an isolated galaxy. In an elaborate statistical study to determine $(M / L)$ in the $V$ band for binary 
galaxies, Schweizer (1987b) found that in general the values for Sc galaxies in interacting pairs range from 12 to 32, with a most likely value of $21 \pm 5$ in solar units. Other works suggest that for this type of galaxy, the value of $(M / L)$ in the $B$ band lies between 10 and 16 (Chengalur et al. 1995; Nordgren et al. 1998; Honma 1999). Most of the determinations of $M / L$ for binary galaxies are based on statistical studies of very large samples; they thus depend on the selection criteria of the latter as well as on the statistical method chosen to probe the $M / L$ of modeled pairs versus observed pairs. It is then of special interest to focus on individual pairs and attempt to determine their $M / L$ ratio through detailed observations and analysis of their kinematics and dynamics. These observations should be complemented by numerical simulations of the encounters varying such parameters as $M / L$ and the structure of the dark matter halo while imposing kinematical restrictions obtained from interferometric 3D observations.

In this work, we present scanning Fabry-Perot observations and the kinematical analysis of the interacting galaxy pair NGC 5426/27 (Arp 271). Section 2 is an overview of the observational parameters and the reduction process. In Sect. 3, a brief bibliographical review of recent works and results on this interacting galaxy pair is presented. In Sect. 4, we present the derived velocity field and the associated rotation curve of each galaxy. The kinematics inferred from the velocity fields and rotation curves are discussed in Sect. 5, giving special attention to the role of non-circular motions. In Sect. 6 the mass of each galaxy is estimated through different methods as well as its mass-to-light ratio. Some suggestions on the structure of the dark matter halo are also presented. In Sect. 7, a possible 3D configuration for the encounter is presented, several features which seem to have been triggered or enhanced by the interaction process are also discussed. Our conclusions are presented in Sect. 8.

\section{Observations}

\subsection{Observations and data reductions}

Observations of NGC 5426/27 (Arp 271) were done on May 1997 at the $\mathrm{f} / 7.5$ Cassegrain focus of the $2.1 \mathrm{~m}$ telescope at the Observatorio Astronómico Nacional in San Pedro Mártir (México), using the scanning Fabry-Perot interferometer PUMA (Rosado et al. 1995).

We used a $1024 \times 1024$ Tektronix CCD detector. In order to enhance the signal, we applied a $2 \times 2$ binning in both spatial dimensions so that the resulting image format was of $512 \times$ 512 pixels with a spatial sampling of $1.18 "$ /pixel. In order to isolate the redshifted $\mathrm{H} \alpha\left(\lambda_{\text {at rest }}=6562.73 \AA\right)$ emission, we used an interference filter centered at $6650 \AA$ with a $F W H M$ of $47 \AA$.

PUMA is a focal reducer built at the Instituto de Astronomía-UNAM used to make direct imagery and Fabry-Perot (FP) spectroscopy of extended emission sources (field of view 10'). The FP used is an ET-50 (Queensgate Instruments) with a servostabilization system having a free spectral range of $19.95 \AA\left(912 \mathrm{~km} \mathrm{~s}^{-1}\right)$ at $\mathrm{H} \alpha$. Its finesse ( 24) leads to a sampling spectral resolution of $0.41 \AA\left(19.0 \mathrm{~km} \mathrm{~s}^{-1}\right)$ which is achieved by scanning the interferometer free spectral range through 48 different channels.

To average the sky variations during the exposure, we got two data cubes with an exposure time of 48 min each $(60 \mathrm{~s}$ per channel). These data cubes were co-added leading to a total exposure time of $96 \mathrm{~min}$. For the calibration we used a $\mathrm{He}$ lamp whose $6678.15 \AA$ line was close to the redshifted nebular wavelength. Two calibration cubes were obtained at the beginning and at the end to check the metrology.

The instrumental and observational parameters are shown in Table 1.

\subsection{Data analysis}

The data reduction and analysis were done using mainly the ADHOCw ${ }^{1}$ software and the CIGALE software (LeCoarer et al. 1993). Standard corrections were done on each of the cubes: removal of cosmic rays, bias and dark subtraction, and flat-fielding. Once the object cubes were co-added, the night sky continuum and $\mathrm{OH}$ sky lines (6627.2 $\mathrm{A}$ and $6634.2 \AA$ ) were subtracted. A spectral Gaussian smoothing $\left(\sigma=57 \mathrm{~km} \mathrm{~s}^{-1}\right)$ was also performed. Once the spectral smoothing was done, the calibration in wavelength was fixed for each profile at each pixel using the calibration cube.

Through the scanning process, we obtained for each pixel a flux value at each of the 48 scanning steps. The intensity profile found along the scanning process contains information about the monochromatic emission $(\mathrm{H} \alpha)$ and the continuum emission of the object. The continuum image computation was done considering the mean of the 3 lowest intensities of the 48 channels cube. For the monochromatic image, the $\mathrm{H} \alpha$ line intensity was obtained by integrating the monochromatic profile in each pixel. The velocity maps were computed using the barycenter of the $\mathrm{H} \alpha$ profile peaks at each pixel. To get a sufficient signal-to-noise ratio on the outer parts of each galaxy, we performed three spatial Gaussian smoothings $\left(\sigma=2.36,3.54,4.72^{\prime \prime}\right)$ on the resulting calibrated cube. A variable-resolution radial velocity map was built using high spatial resolution (less spatially-smoothed pixels) for regions with originally higher signal-to-noise ratio. No double peaks appeared in the velocity profiles.

The profiles were deconvolved by the instrumental profile. The $F W H M$ for each pixel was computed from the deconvolved profile at each pixel. For each pixel, the threshold of the profile was defined by the value of the channel for which $60 \%$ of the channels had a larger intensity value.

\section{NGC 5426 and NGC 5427}

Arp 271 is an interacting pair consisting of two spiral galaxies, NGC 5426 and NGC 5427, of approximately the same angular size (2.3') (Fig. 1). This pair was first catalogued by VorontsovVelyaminov (1959). It was later included in Arp's Atlas of Peculiar Galaxies (Arp 1966) grouped with other objects he

\footnotetext{
${ }^{1}$ http://www.oamp.fr/adhoc/adhocw.htm developed by J. Boulesteix.
} 


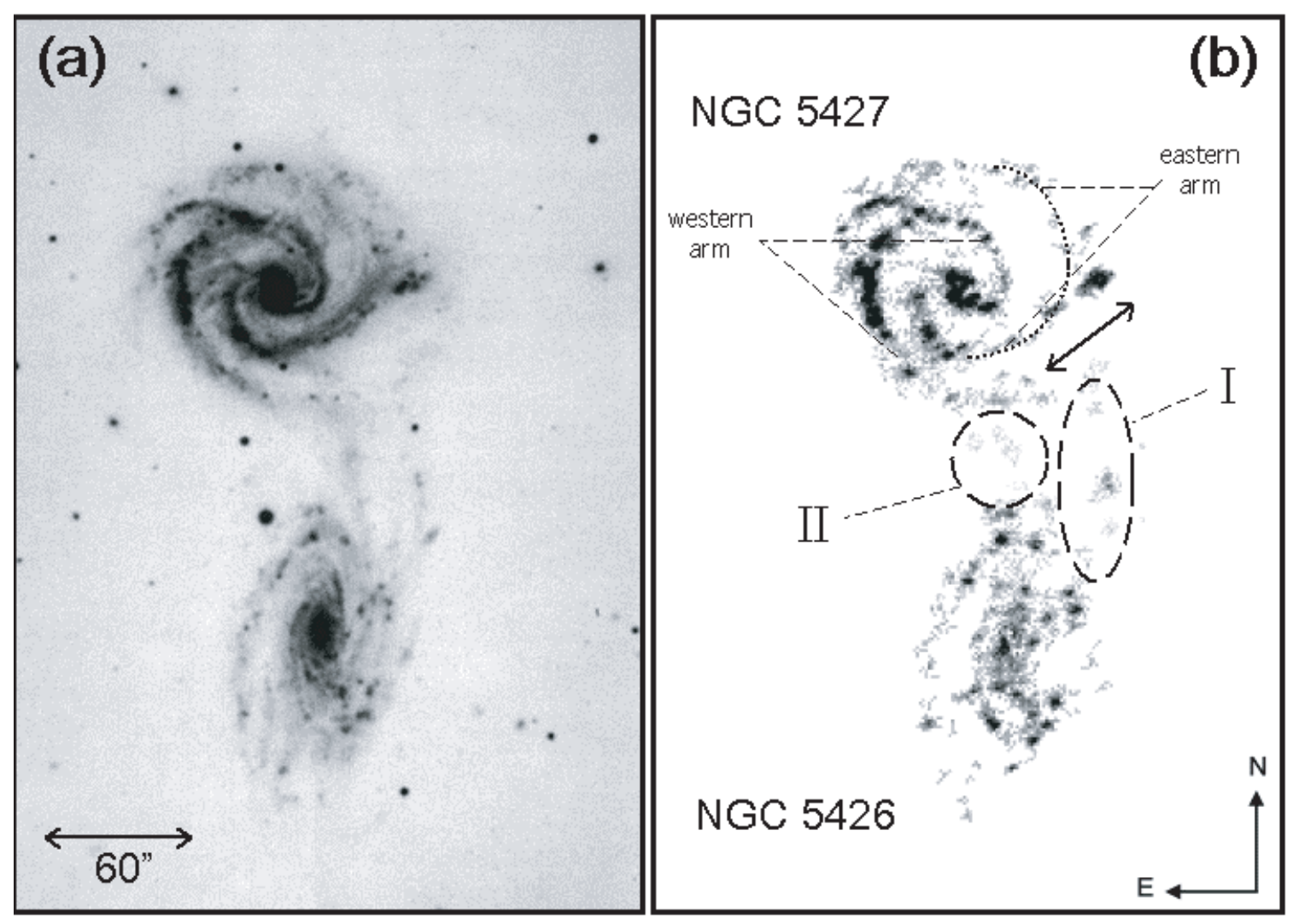

Fig. 1. a) Direct $B$ image of NGC 5426/27 (Arp 271) taken from "The Carnegie Atlas of Galaxies. Volume II" (Sandage \& Bedke 1994). b) Monochromatic $\mathrm{H} \alpha$ (continuum substracted) image of the pair obtained with the scanning Fabry-Perot interferometer PUMA data cubes (see text for reduction process). Arrow indicates straight-arm segment, dotted line traces what would be a "classical" spiral arm. Regions I and II are associated to the bridge-like feature of gas between both galaxies.

classified as double galaxies with connecting arms. This connection is clearly visible in the ultra-high contrast $J$-band image of the pair presented by Blackman (1982).

Several works have been done on this pair of galaxies, mostly as part of vast surveys of interacting galaxies. In an extensive analysis of morphological correlation for over 16000 nearest neighbor pairs of galaxies, Yamagata et al. (1989) found that NGC 5426 and NGC 5427 not only have the same morphological type but also very similar internal structure as well as comparable sizes. These characteristics make Arp 271 fall into the category of twin galaxies proposed by these authors. According to them, this type of paired galaxies suggest the possibility of fission processes (Page 1975) at the origin of the present morphological type of galaxies since it is easy to understand this morphological resemblance if the galaxies considered formed under the same initial conditions given by the proximity of their "places of birth". Blackman (1982) found an asymmetric distribution of the light of both galaxies: $38 \%$ of the total flux comes from the two adjacent halves of the galaxies. According to him, this dimming of coincidental sides of binary galaxies is observed in other interacting pairs (Arp 1966) and can be due to the time scale of the interaction (not large enough to average the density of perturbed material and subsequent star formation) and/or to obscuration of one side of one of the galaxies by the other galaxy.
NGC 5426 is a type SA(s)c pec (de Vaucouleurs et al. 1991) and luminosity class II (Blackman 1982). A heliocentric systemic velocity of $2584 \mathrm{~km} \mathrm{~s}^{-1}$ was found through long-slit spectroscopical observations (Schweizer 1987a). From photometric observations, long-slit spectroscopy and using the method established by Nordsieck (1973), Blackman (1982) found a mass-to-light ratio of $(M / L)_{V}=4.1 \pm 0.6$ and $(M / L)_{B}=3.9 \pm 0.7$ within a radius of $22.5 \mathrm{kpc}$. These values include corrections for internal absorption. NGC 5427 is a $\mathrm{SB}(\mathrm{s}) \mathrm{c}$ pec, Seyfert 2 galaxy with luminosity class I (de Vaucouleurs et al. 1991). Through tertiary indicators, it is located at 26.7 Mpc (de Vaucouleurs 1979) with a systemic heliocentric velocity of $2730 \mathrm{~km} \mathrm{~s}^{-1}$ (Schweizer 1987a). Up to a radius of $14.5 \mathrm{kpc}$, this galaxy has $(M / L)_{V}=2.2 \pm 0.3$ and $(M / L)_{B}=1.7 \pm 0.2$ (Blackman 1982). González-Delgado \& Pérez (1992) find a high rate of star formation in the galaxy disk, as well as a higher number of HII regions (both giant and supergiant) than expected for its morphological type. Alfaro et al. (2001) obtained the rotation curve of this galaxy through long-slit spectroscopy along its major axis. They identified several oscillations along this curve that seem to be correlated to the presence of spiral arms and suggest taht this behavior is similar to the one expected in a galactic "bore" (or hole) generated by the interaction of a spiral density wave with a thick gaseous disk. Table 2 lists the main parameters of each galaxy. 
Table 2. Parameters of NGC 5426 and NGC 5427.

\begin{tabular}{|c|c|c|}
\hline & NGC 5426 & NGC 5427 \\
\hline \multirow[t]{2}{*}{ Coordinates $(\mathrm{J} 2000)^{a}$} & $\alpha=14 \mathrm{~h} 03 \mathrm{~m} \mathrm{24.8s}$ & $\alpha=14 \mathrm{~h} 03 \mathrm{~m} 26.0 \mathrm{~s}$ \\
\hline & $\delta=-06^{\circ} 04^{\prime} 09^{\prime \prime}$ & $\delta=-06^{\circ} 01^{\prime} 51^{\prime \prime}$ \\
\hline Morphological type ${ }^{b}$ & $\mathrm{SA}(\mathrm{s}) \mathrm{c}$ pec & SA(s)c pec Sey 2 \\
\hline Luminosity class $^{c}$ & II & I \\
\hline$m_{B}^{d}(\mathrm{mag})$ & 12.86 & 12.06 \\
\hline$D_{25} / 2^{d}\left({ }^{\prime}\right)$ & 1.475 & 1.435 \\
\hline $\operatorname{Distance}^{e}(\mathrm{Mpc})$ & --- & 26.7 \\
\hline$M_{B}^{d}(\mathrm{mag})$ & -20.567 & -21.167 \\
\hline Average surface brightness within $D_{25} / 2^{d}$ (in mag $/ /^{\prime 2}$ ) & 23.50 & 22.86 \\
\hline \multirow[t]{3}{*}{ Systemic velocity $\left(\mathrm{km} \mathrm{s}^{-1}\right)$} & $2516 \pm 5^{c}$ & $2703 \pm 20^{c}$ \\
\hline & $2584 \pm 9^{f}$ & $2730 \pm 9^{f}$ \\
\hline & $2575 \pm 3^{g}$ & $2722.5 \pm 1^{g}$ \\
\hline \multirow[t]{2}{*}{$V_{\text {rot } \max }\left(\mathrm{km} \mathrm{s}^{-1}\right)$} & $209^{h}$ & $172^{h}$ \\
\hline & $200^{i}$ & --- \\
\hline \multirow[t]{2}{*}{$\mathrm{PA}\left({ }^{\circ}\right)$} & $180^{c}$ & $68^{c}$ \\
\hline & $177.5 \pm 1^{g}$ & $53.2 \pm 3^{g}$ \\
\hline \multirow[t]{3}{*}{ Inclination $\left({ }^{\circ}\right)$} & $59 \pm 2^{c}$ & $32 \pm 2^{c}$ \\
\hline & - - - & $24.49^{j}$ \\
\hline & $59 \pm 3^{g}$ & $34 \pm 2.5^{g}$ \\
\hline \multirow[t]{2}{*}{ Mass (in $10^{10} M_{\odot}$ ) } & $3.39 \pm 0.13^{c, k}$ & $3.1 \pm 0.4^{c, l}$ \\
\hline & $11.2^{g, m}$ & $7.5^{g, m}$ \\
\hline
\end{tabular}

a NED database.

${ }^{b}$ de Vaucouleurs et al. (1991).

c Blackman (1982).

${ }^{d}$ LEDA database.

$e$ Through tertiary indicators (de Vaucouleurs 1979).

$f$ Schweizer (1987a).

$g$ This work.

${ }^{h}$ From $\mathrm{H} \alpha$ rotation curve - this work.

From $21 \mathrm{~cm}$ observations (Bottinelli et al. 1948).

Keel 1996.

${ }^{k}$ Inside $7.8 \mathrm{kpc}=0.84 D_{25} / 2$ using method of Nordsieck (1973).

${ }^{l}$ Inside $9.4 \mathrm{kpc}=0.68 D_{25} / 2$ using method of Nordsieck (1973).

${ }^{m}$ Inside $D_{25} / 2$ considering a spheroidal distribution.

\section{Velocity fields and rotation curves}

\subsection{Monochromatic image}

Figure $1 \mathrm{~b}$ displays the monochromatic $\mathrm{H} \alpha$ image of the pair. The HII regions of NGC 5426 are rather knotty and they are mainly found in the western half of the galaxy. The eastern and southeastern parts of the galaxy are practically devoid of any important HII regions. For NGC 5427 most of the HII-regions are located to the north-eastern side of the galaxy along the western arm forming a rather patchy structure. Many strong HII-regions appear also at the beginning of the eastern arm, but become less intense at the point where a straight arm segment begins. At the end of the straight arm there is a very bright HII-region, not following the logarithmic spiral arm. The intensity of the emission decreases once the logarithmical pattern is continued. We detect most of the HII regions detected by González-Delgado \& Pérez (1992). A central bar of about 32" in total length is also detected on the monochromatic image of this galaxy.

Patches of faint monochromatic emission can be traced between both galaxies outlining a bridge of ionized gas between both members of the pair. This bridge follows approximately the same orientation as the bridge of stars (continuum) that can be seen in both the direct image of the pair (Fig. 1a) and the ultracontrast $J$-band image from Blackman (1982). It seems to be divided into two narrow monochromatic filaments: I and II (Fig. 1b). Filament I could be interpreted as an extension of the western arm of NGC 5426, while the location of 


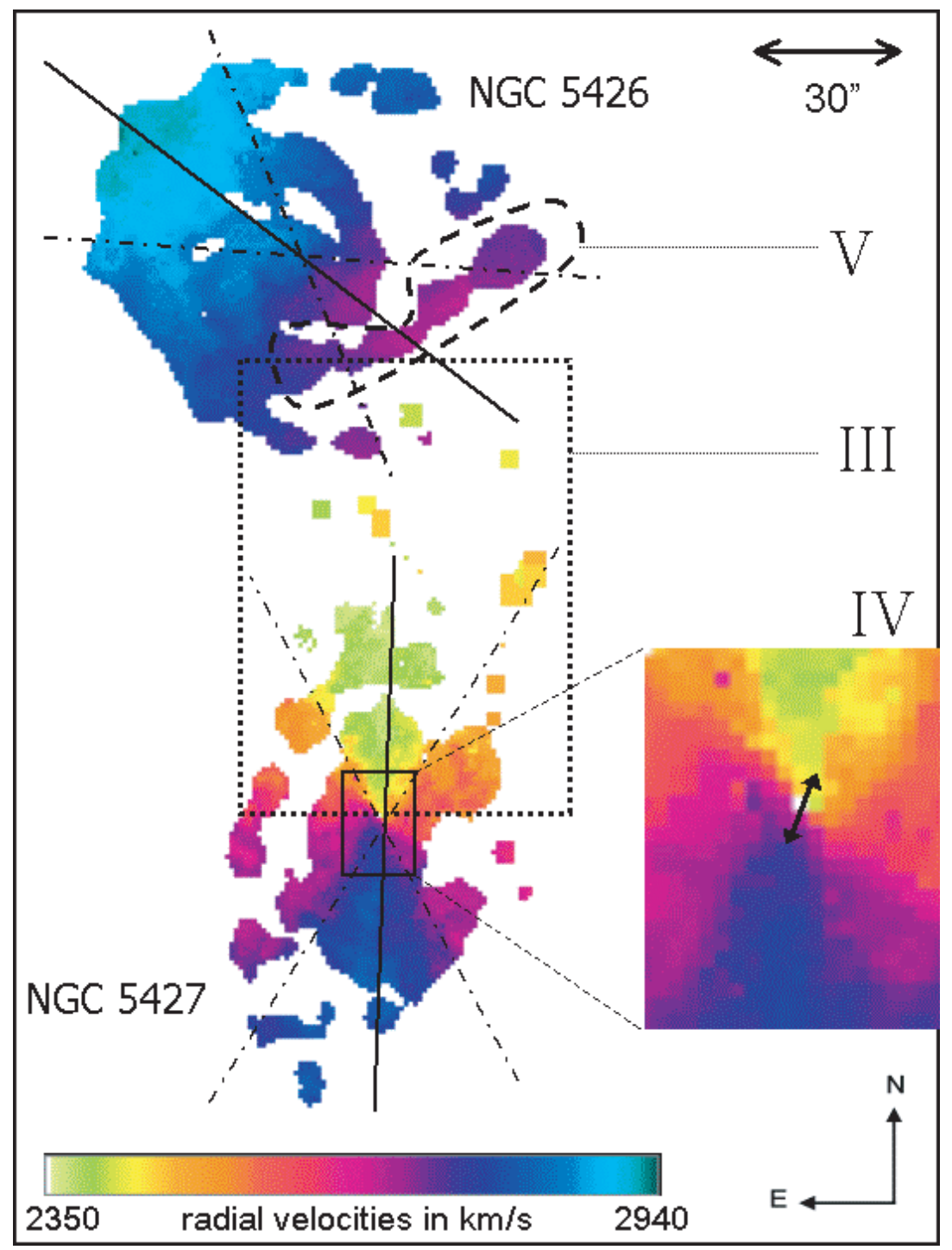

Fig. 2. Velocity fields of NGC 5426 and NGC 5427. Solid lines indicate each galaxy's position angle (PA), slash-dotted lines indicate the angular sectors from both sides of the major axis considered for the computation of each galaxy's rotation curve. Region III covers the bridgelike feature between both galaxies, region IV (enlarged) is associated with the central parts of NGC 5426 where a small bar-like feature is outlined. Arrow in the enlargement indicates the location and size $\left(6^{\prime \prime}\right)$ of a small bar-like feature outlined by NGC 5426's isovelocities. Region V indicates the straight-arm segment in NGC 5427.

filament II makes it harder to associate this feature with any of the galaxies' arms.

\subsection{Velocity fields}

Figure 2 shows the velocity fields of NGC 5426/27. Figure 3 shows the associated isovelocities.

For the main body of the galaxy, the velocity field of NGC 5426 shows little distortion if compared to the velocity field of an isolated disk galaxy, though the zero-velocity line of the galaxy shows large wiggles. The northern and southern parts (approaching and receding sides, respectively) are rather symmetric. In the outer eastern parts, the isovelocities no longer follow the classical "V-pattern" of the central parts but instead become almost horizontal. This part of the velocity field corresponds to one of the galaxy's spiral arms. In the central parts of the field (inner $6^{\prime \prime}$ ), we notice a small shift with respect to the kinematical center between the isovelocities of the receding and approaching sides (marked with an arrow in region IV, Fig. 2). In this region, the isovelocities are almost parallel to each other following a southwestern-northeastern direction along a distance of about 6" (Fig. 3). This behaviour which can be related to a rigid-body-like rotation, could be associated to the presence of an incipient small bar-like feature perpendicular to these isovelocities and of a length of about $66^{\prime \prime}$.

For NGC 5427, the isovelocities on the approaching (SW) and receding (NE) sides are more opened and patchy than the ones for NGC 5426. The zero-velocity line of this galaxy also shows large wiggles mostly in the outer parts of the galaxy. The isovelocities on the western side (closest to NGC 5426) show no clear orientation. More than one third of the straight arm has a mean radial velocity of $(2652 \pm 7) \mathrm{km} \mathrm{s}^{-1}$ while other third 


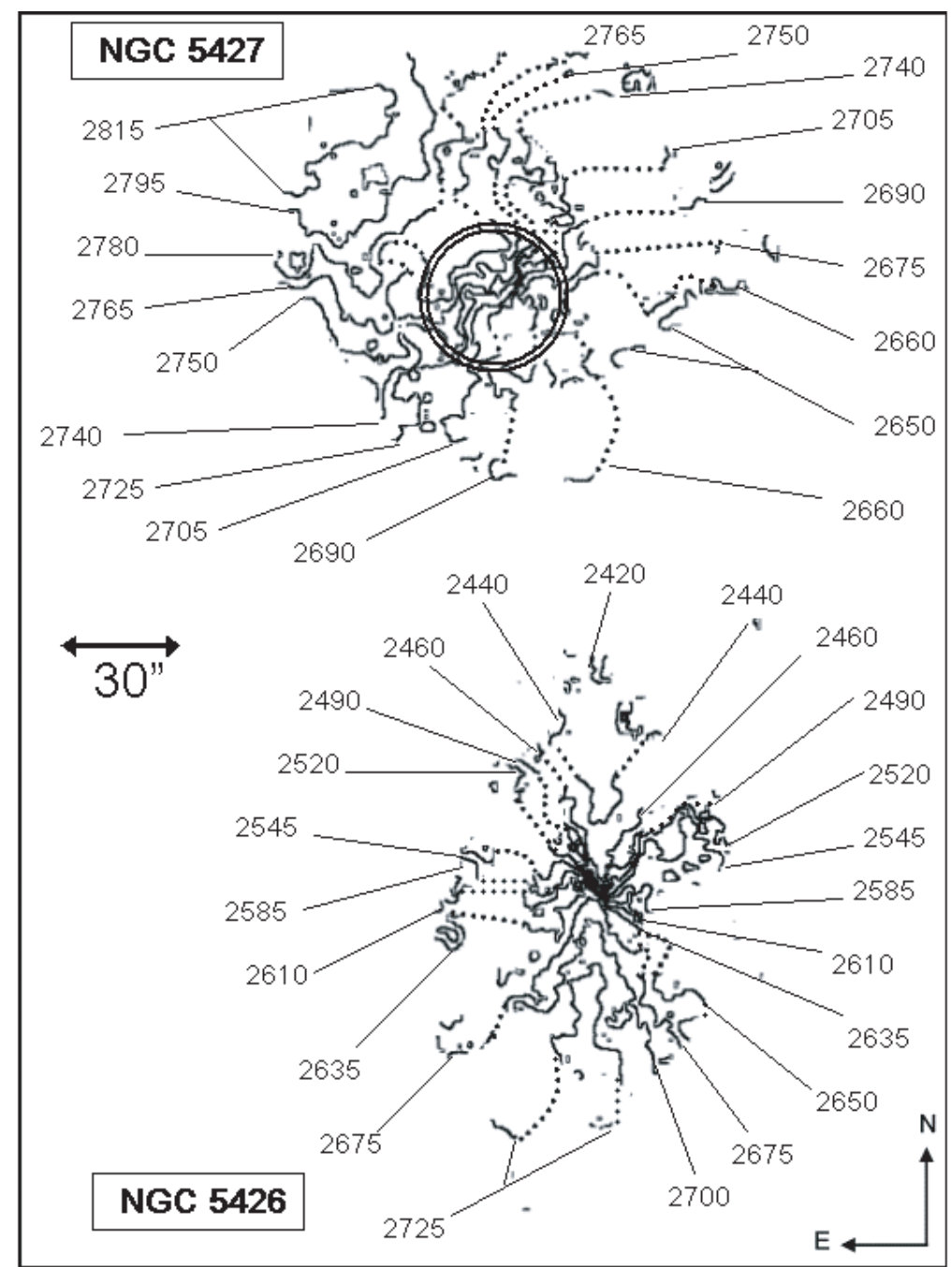

Fig. 3. Isovelocities of NGC 5426 and NGC 5427. The circled region shows central bar in NGC 5427. Velocities displayed are in $\mathrm{km} \mathrm{s}^{-1}$. Dotted lines are eye-estimated interpolation of the data. Systemic velocities are $2575 \pm 3 \mathrm{~km} \mathrm{~s}^{-1}$ for NGC 5426 and $2722.5 \pm 1 \mathrm{~km} \mathrm{~s}^{-1}$ for NGC 5427 .

has a different mean value of $(2682 \pm 7) \mathrm{km} \mathrm{s}^{-1}$ (Region $\mathrm{V}$ in Fig. 2). This velocity distribution differs considerably from the isovelocities observed on the other arm of NGC 5427 (western arm and its bifurcation) where the range of observed radial velocities is much broader. In the central parts rather parallel isovelocities are found within the inner $30 \pm 2^{\prime \prime}$ (circled region in Fig. 3). These isovelocities outline the central bar visible in the monochromatic image.

The radial velocities associated to the HII regions outlining the bridge between both galaxies (regions I and II in Fig. 1b) are enlarged in Fig. 4. Most of these regions have radial velocities closer to those of NGC 5426 (regions A to L) and are furthermore suspected to belong to this galaxy. Only regions on the northeastern part of the bridge have radial velocities closer to those of NGC 5427 (regions M and N) and probably belong to the spiral arm of NGC 5427.

\subsection{Rotation curves}

The interacting process perturbs each of the galaxies involved in the encounter and introduces an important contribution of non-circular movements in the velocity fields. Nevertheless, in the case of early-stage interactions, one can assume that the inner parts of galaxies are not strongly perturbed, so that the velocity fields are still smooth and symmetrical resulting in symmetric and low-scattered rotation curves up to a certain radius.

With this assumption in mind, the rotation curve of each galaxy was computed considering different values for the kinematical parameters involved (heliocentric systemic velocity $V_{\text {syst }}$, kinematical center, inclination $i$ and position angle PA). The values were chosen such to obtain a symmetric curve in the inner parts of the galaxy and to minimize scatter on each side of the curve - for further detail on the computation of the rotation curve see Amram et al. (1996). The first step to reduce this scatter was to consider points on the velocity field within a certain angular sector on each side of the galaxy's major axis. The value of $i$ was then computed from the ratio between the major and minor axis and the PA from the orientation of the latter. If a single ellipse was difficult to adjust to the continuum image, we considered an ellipse whose semi-minor axis corresponded to the kinematical semi-minor axis on the velocity field of the galaxy and whose semi-major axis was given 


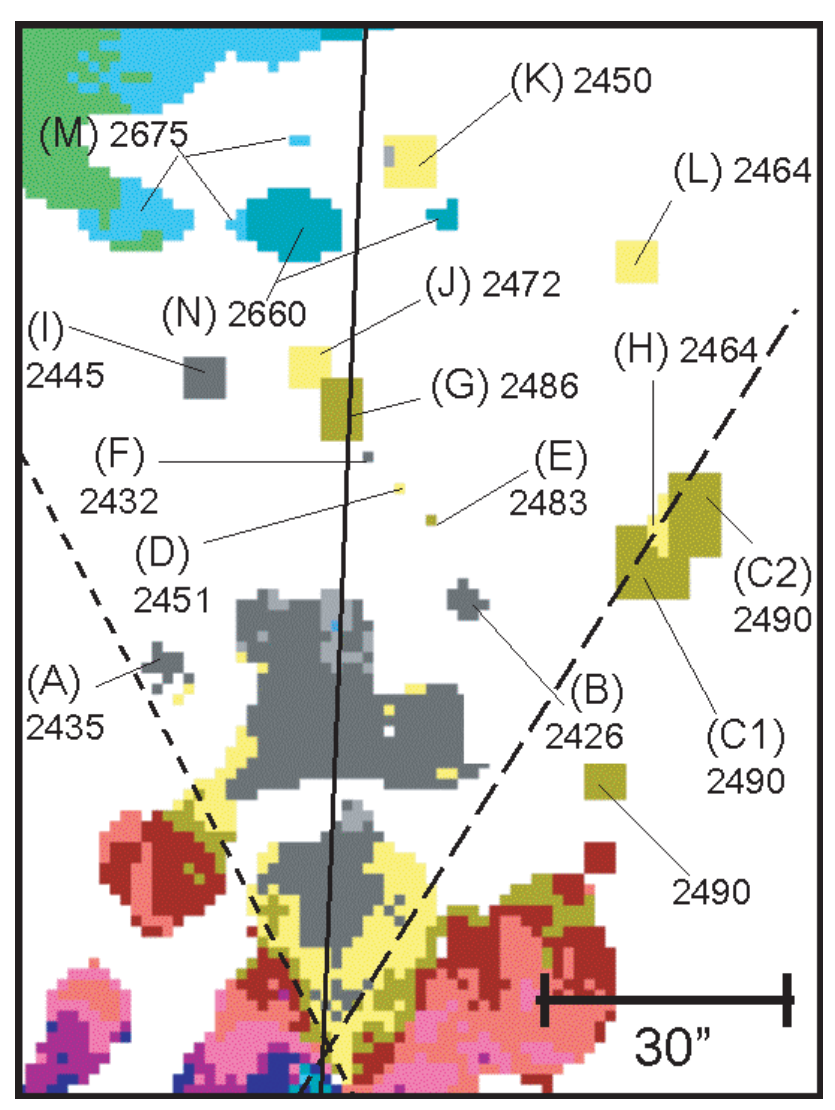

Fig. 4. Velocity field of the bridge-like H $\alpha$ feature between NGC 5426 and NGC 5427. Letters are related to particular kinematical features in NGC 5426's rotation curve shown in Fig. 5.

by the furthest emission point on the continuum image along a line perpendicular to the semi-minor kinematical axis.

\subsubsection{NGC 5426}

The rotation curve of NGC 5426 was computed considering points on the velocity field within an angular sector of $30^{\circ}$ on each side of the galaxy's PA. For this galaxy, the kinematical center used to compute the rotation curve matches the photometric center within $1^{\prime \prime}$. Within $60^{\prime \prime}$, the set of values that resulted in the most symmetrical, smooth and less-scattered curve was $\mathrm{PA}=(177.5 \pm 1)^{\circ}, i=(59 \pm 3)^{\circ}$ and $V_{\text {sys }}=(2575 \pm$ $3) \mathrm{km} \mathrm{s}^{-1}$. These are presented in Table 2 . Figure $5 \mathrm{~b}$ shows that the rotation curve is rather symmetrical in its inner $60^{\prime \prime}$. After the steep solid-body rotation curve at $R<3.5^{\prime \prime}$, it rises slowly from $130 \mathrm{~km} \mathrm{~s}^{-1}$ to $200 \mathrm{~km} \mathrm{~s}^{-1}$ at $R=65^{\prime \prime}$. The rotation curve shows slight oscillations in phase on both sides. For the receding side (southern side, opposite from the companion), the rotation curve keeps rising with smooth oscillations up to the last detected emission point at $R=80^{\prime \prime}$. At this radius, the rotational velocity reaches its maximum value $V_{\max }=209 \mathrm{~km} \mathrm{~s}^{-1}$ close to $200 \mathrm{~km} \mathrm{~s}^{-1}$ found with HI observations (Bottinelli et al. 1984). For the approaching side (north side, towards the companion), at $R=65^{\prime \prime}$ the rotational velocity abruptly falls to a value of $(128 \pm 12) \mathrm{km} \mathrm{s}^{-1}$. Between $62^{\prime \prime}$ and $80^{\prime \prime}$, the rotational velocity drops to $\sim 100 \mathrm{~km} \mathrm{~s}^{-1}$. From $R=82^{\prime \prime}$, an average value of $150 \mathrm{~km} \mathrm{~s}^{-1}$ is kept up to $R=110^{\prime \prime}$. Comparing with previous values, our value for $V_{\text {syst }}$ is similar to the one found by Schweizer (1987a), nevertheless there is an important difference with the value of the $V_{\text {syst }}$ determined by Blackman (1982). To compare our rotation curve with the one obtained by Blackman (1982), a long-slit was simulated by considering radial velocities on the velocity field within a sector of $1^{\circ}$ on each side of PA. We then had to correct Blackman's curve by the inclination of the disk in order to superpose both curves. The shape and amplitude of both rotation curves are very similar up to 50" (Fig. 6).

\subsubsection{NGC 5427}

An angular sector of $32^{\circ}$ on each side of the galaxy's PA was considered for the computation of the rotation curve of NGC 5427 in order to reduce asymmetry and scatter up to a radius of $47^{\prime \prime}$. Since more than a single ellipse could be adjusted to the irregular outermost isophote of the continuum image, we considered an ellipse whose semi-minor axis corresponded to the kinematical semi-minor axis on the velocity field of the galaxy (radial velocities corresponding to $\sim 2740 \mathrm{~km} \mathrm{~s}^{-1}$ in Fig. 3) to compute the inclination $i$ and the PA of this galaxy. The semi-major axis of the resulting ellipse was then given by the furthest emission point on the continuum image along a line perpendicular to the semi-minor kinematical axis. This perpendicular line was taken as the direction of the PA. The set of values that resulted in the most symmetrical, smooth and lessscattered curve was PA $=(53.2 \pm 3)^{\circ}, i=(34 \pm 2.5)^{\circ}$ and $V_{\text {sys }}=(2722.50 \pm 1) \mathrm{km} \mathrm{s}^{-1}$ (Table 2). The kinematical center used to compute the rotation curve of NGC 5427 matches the photometric one within $1^{\prime \prime}$. Globally the rotation curve displays an ascending behavior reaching $V_{\text {rot }}=154 \mathrm{~km} \mathrm{~s}^{-1}$ at $R=46.5^{\prime \prime}$ (Fig. 7). There is however a trough on both sides of the curve between $18^{\prime \prime}$ and $29^{\prime \prime}$. Up to $R=46.5^{\prime \prime}$, the curve is rather symmetric. After this point, the radial velocities on the approaching side (south to the companion) decrease by an abrupt shift of $50 \mathrm{~km} \mathrm{~s}^{-1}$ with respect to the receding side. On the receding side, the curve increases up to $V_{\text {rot }}=172 \mathrm{~km} \mathrm{~s}^{-1}$. From $R=51^{\prime \prime}$ onwards the curve flattens around a value of $\sim 172 \mathrm{~km} \mathrm{~s}^{-1}$ up to the last emission point on this side of the galaxy at $62^{\prime \prime}$. Our resulting rotation curve resembles the one found by Blackman (1982), both in its general form as well as in the maximum rotational velocity $\sim 175 \mathrm{~km} \mathrm{~s}^{-1}$. In order to compare our rotation curve to the rotation curve obtained by Alfaro et al. (2001), a long-slit was simulated on our observed velocity field along the same position as these authors and corrected with their inclination and PA $\left(30^{\circ}\right.$ and $70^{\circ}$, respectively) finding the same oscillating behavior on both sides of the galaxy (Fig. 8). $V_{\text {syst }}$ by Blackman (1982), Schweizer (1987a) and Keel (1996) are in good agreement with ours. For the inclination $i$ and the PA, important differences are found between the values determined in different works. Our values are larger than those found by Keel (1996) and Blackman (1982) who also used kinematical considerations - see Table 2. For NGC 5427, the inclination determination in our case is partly based on isophotal shape which makes it rather uncertain since for inclinations of $\sim 30^{\circ}$, the deviations from circular shape are 

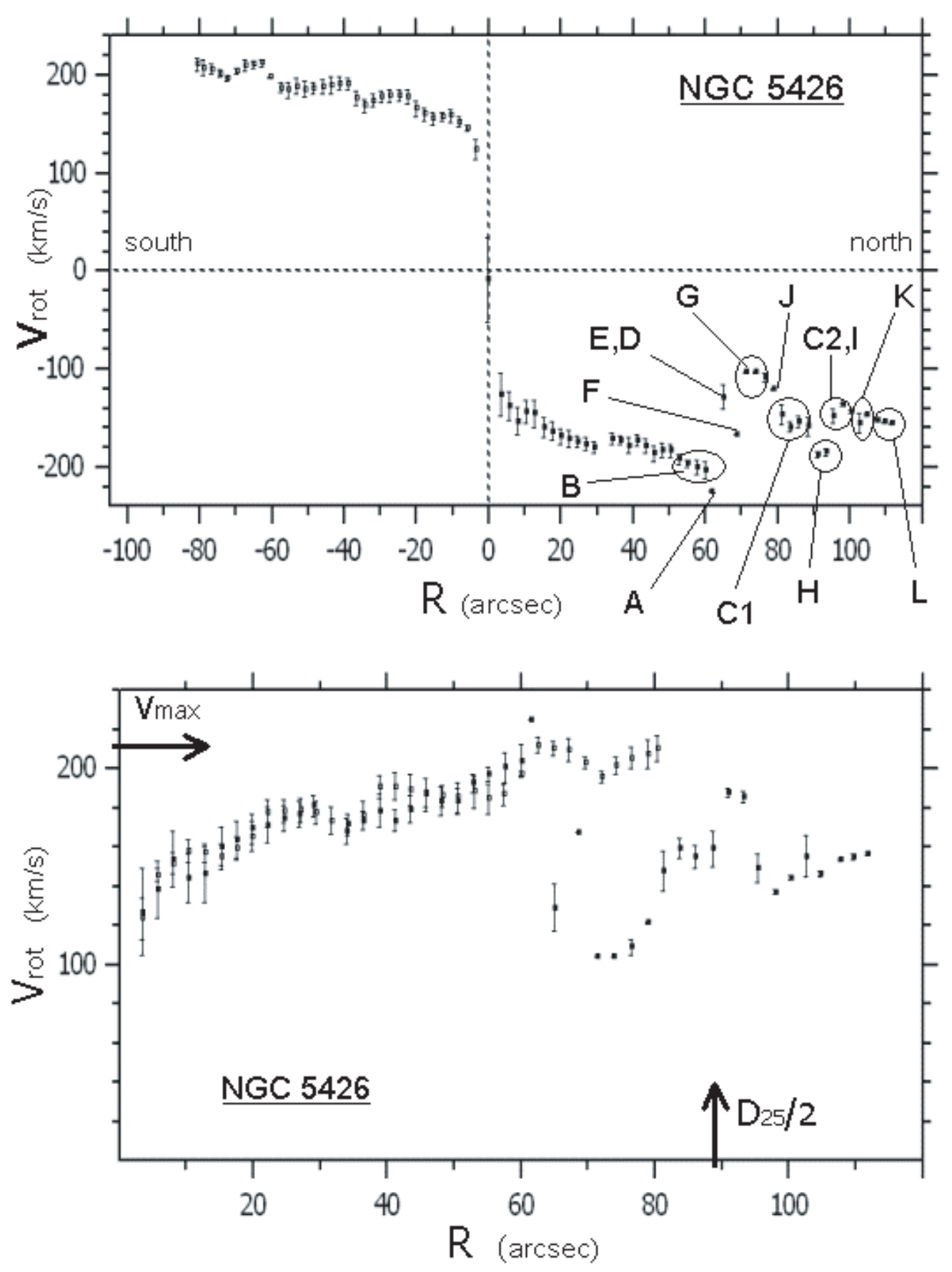

Fig. 5. Rotation curve of NGC 5426. Upper panel shows the $V(R)$ vs. $R$ for both sides of the galaxy -approaching and receding side. Letters are associated to the regions shown in Fig. 4. Lower panel shows the superposition of both sides. $V_{\max }$ indicates the rotation velocity value chosen to compute the mass of this galaxy within $D_{25} / 2$ also indicated in this figure.

small $\left(\cos 34^{\circ}=0.83, \cos 24^{\circ}=0.91\right)$ which are hard to distinguish. Plus the orientation effect on isophotal shape might easily be over-shadowed by the effects of spiral arms or mild deformations due to the interaction (any such effect is likely to increase the deduced inclination).

\section{Non-circular motions}

Two dimensional kinematical fields of disk galaxies allow us to study the motion of the gas all over the galaxy and to match it with structures found through photometric methods in order to determine to which extent the gas is following pure circular motion around the center of the galaxy and to which extent there are important contributions from non-circular velocities (radial and azimuthal).

\subsection{Full width at half maximum (FWHM)}

The $F W H M$ of the profile at each pixel was computed from the intensity profile at each pixel as described in Sect. 2.2 


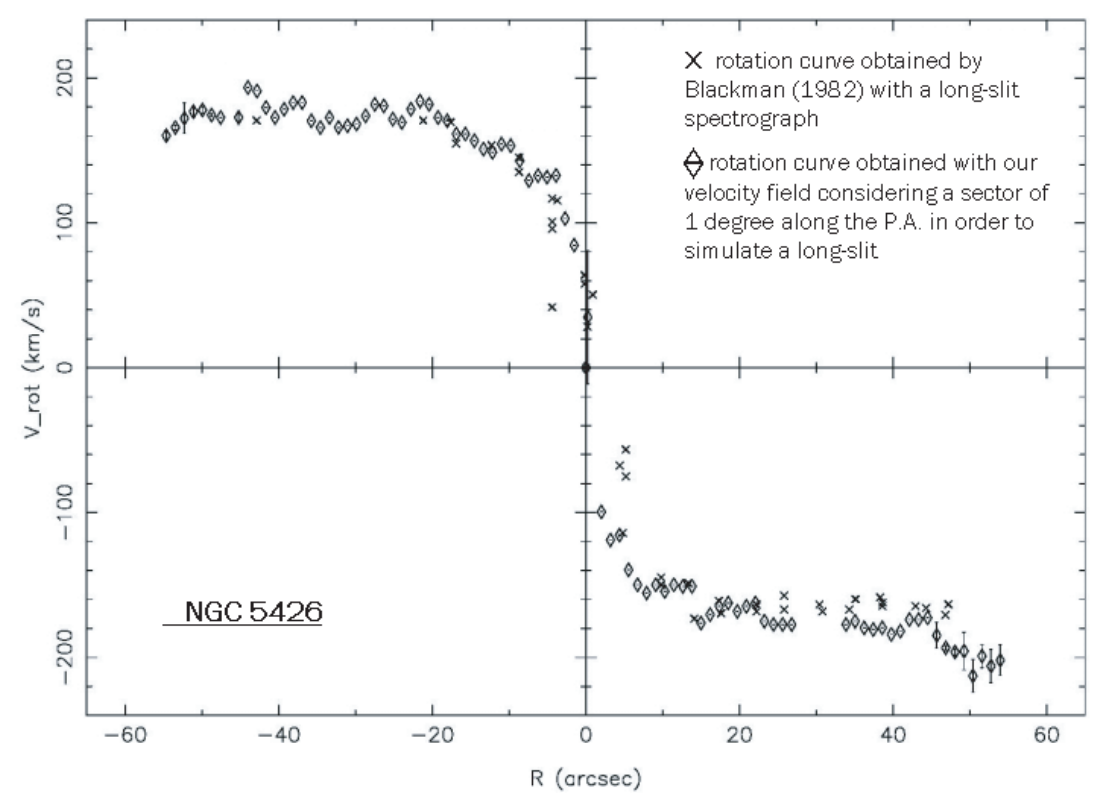

Fig. 6. Comparison between the rotation curve obtained by Blackman (1982) with a long-slit spectrograph (crosses) and our rotation curve obtained by simulating a long-slit spectrograph rotation curve (diamonds) considering the same parameters as that author (PA, $i$, center, angular sector).

Depending on the inclination of the galaxy, this width may be associated to motions of the gas perpendicular to the plane of the galaxy (in the case where the galaxy is almost face-on, $i \sim 0^{\circ}$ ) or to non-circular motions in the plane of the galaxy (if the galaxy is almost edge-on, $i \sim 90^{\circ}$ ). For intermediate values of $i$, the $F W H M$ is associated to both types of motion. FWHM fields for each galaxy are shown in Fig. 9.

\subsubsection{NGC 5426}

The central HII regions of this galaxy display important values of $F W H M\left(>140 \mathrm{~km} \mathrm{~s}^{-1}\right)$. However the amplitude of $F W H M$ is not simply correlated to the flux of these HII regions. Large FWHM amplitudes $\left(\sim 170 \mathrm{~km} \mathrm{~s}^{-1}\right)$ are slightly shifted to less intense HII regions. They match the extremities of the small bar-like feature outlined in the velocity field and thus could be associated with the motion of gas due to the presence of this incipient bar. A peculiar $\sim 35^{\prime \prime}$ cross-like pattern (NE-SW and SE-NW) of large $F W H M$ appears in the main body of the galaxy (region A in Fig. 9a). It is not associated with any particular morphological feature in the monochromatic image but it does coincide with the orientation of the radial isovelocities in this part of the galaxy. Large $F W H M\left(>140 \mathrm{~km} \mathrm{~s}^{-1}\right)$ are also found along the spiral arm of the galaxy (regions B, C and D in Fig. 9a).

\subsubsection{NGC 5427}

Large $F W H M$ values are found in the central regions of NGC 5427 matching the maximum of the monochromatic image and could be associated to the star-forming processes in these regions. Nevertheless they do not follow the orientation of the central bar. In general there seems to be a trend in NGC 5427 showing that the FWHM increases toward the faint edges of the spiral arms, both in the eastern and in the western arms, at least in those parts of the spiral arms where the arms are strong. The northeastern and eastern parts of the galaxy show regions with larger $F W H M$ are seen (regions E and $\mathrm{F}$ in Fig. 9b). These regions do not coincide with any HII region, on the contrary, they lie on each side of this galaxy's western spiral arm where emission decreases considerably. In particular region $\mathrm{E}$ falls on the region observed by Alfaro et al. (2001) with a long-slit spectrograph and where oscillations on their rotation curve are interpreted as gas being decelerated as it enters the spiral arm and jumping above the arm to land on a region on the other side of the arm. Since NGC 5427 has a low inclination $\left(i=34^{\circ}\right)$, the large $F W H M$ observed in regions $\mathrm{E}$ and $\mathrm{F}$ can actually be associated to the perpendicular motion of the gas before and after it encounters the spiral arm as described by these authors.

\subsection{Residual velocity fields}

Residual velocity fields of both galaxies were obtained by subtracting the observed radial velocity field from a ideal radial velocity field. The ideal field is the radial velocity field of an ideal galaxy in which there are only uniform circular motions in a plane so that

$V_{\text {ideal }}(R)=V_{\text {syst }}+\left(V_{\text {circ }}(R) \times \cos \theta \times \sin i\right)$

where $V_{\text {syst }}$ is the systemic velocity of the galaxy, $V_{\text {cir }}(R)$ is the circular velocity component at a radius $R, \theta$ is the angle along the galaxy plane, counted from the approaching side of the nodal line and increasing in the direction of the galaxy 

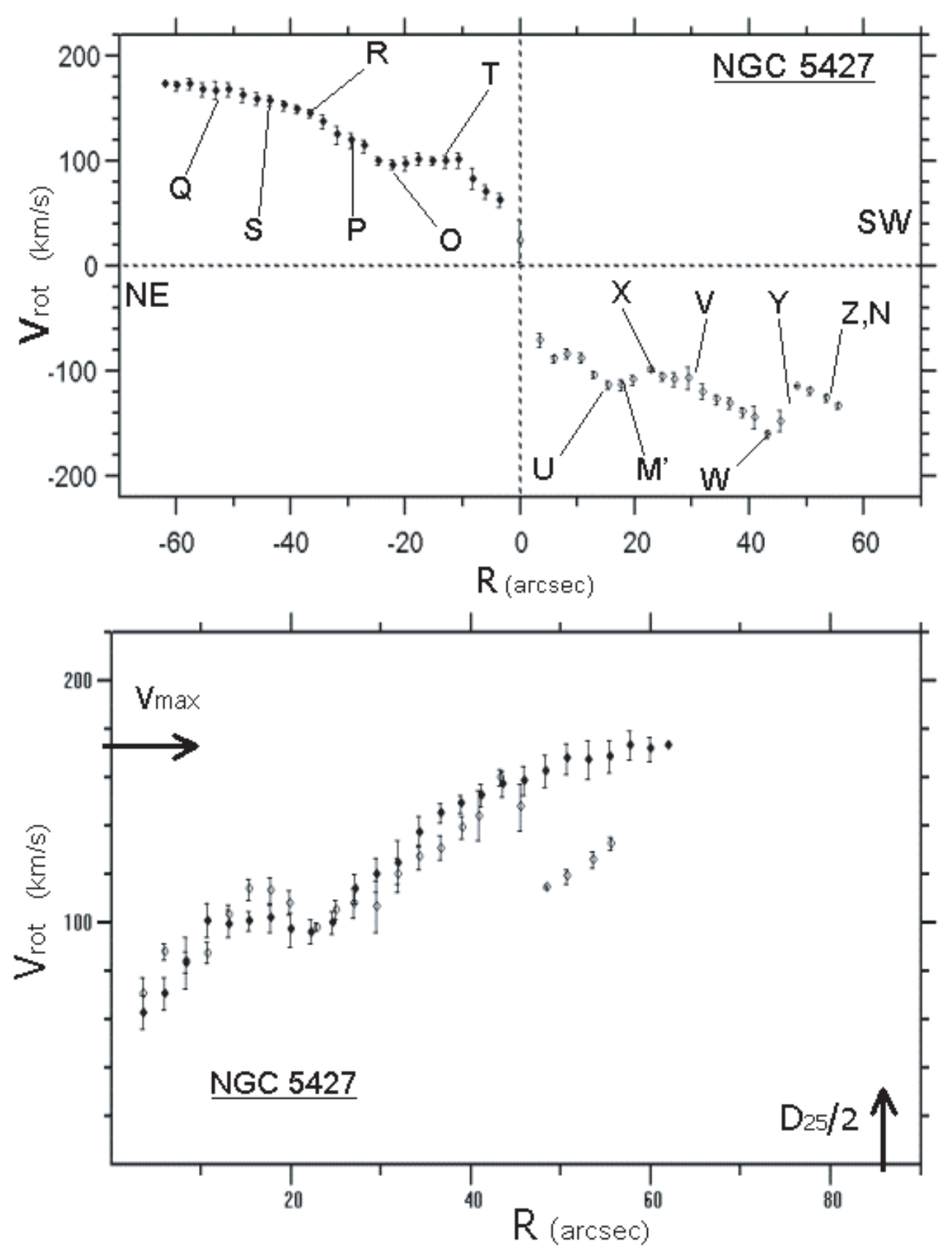

Fig. 7. Rotation curve of NGC 5427. Upper panel shows the $V(R)$ vs. $R$ for both sides of the galaxy - approaching and receding side. Letters are associated to the regions shown in Fig. 12. Lower panel shows the superposition of both sides. $V_{\max }$ indicates the rotation velocity value chosen to compute the mass of this galaxy within $D_{25} / 2$ also indicated on this figure.

rotation and $i$ is the galaxy inclination with respect to the plane of the sky.

The ideal velocity field is constructed from the observed rotation curve by assigning to all the points of the galaxy at a certain radius, the rotational velocity from the rotation curve at that particular radius. These fields prove to be very useful to evaluate the validity of the kinematical parameters chosen to compute the rotation curve of a disk galaxy (Warner et al. 1973). They also give the opportunity to detect and analyze non-circular motions of the gas in each galaxy since points where the residual velocity $V_{\text {res }} \sim 0$ correspond to regions in the galaxy where circular motions predominate. For points where $V_{\text {res }}>0$ or $V_{\text {res }}<0$, the interpretation becomes more complicated. In the general case, the observed radial velocity $V_{\text {obs }}$ has the following decomposition:

$$
\begin{aligned}
V_{\mathrm{obs}}= & V_{\mathrm{syst}}+\left[\left(V_{\mathrm{circ}}+V_{\mathrm{tan}}\right) \times \cos \theta+V_{\mathrm{rad}} \times \sin \theta\right] \\
& \times \sin i+V_{\perp} \times \cos i
\end{aligned}
$$

where $V_{\tan }$ is the tangential velocity component in the plane of the galaxy (additional to the circular velocity component $V_{\text {cir }}$ 


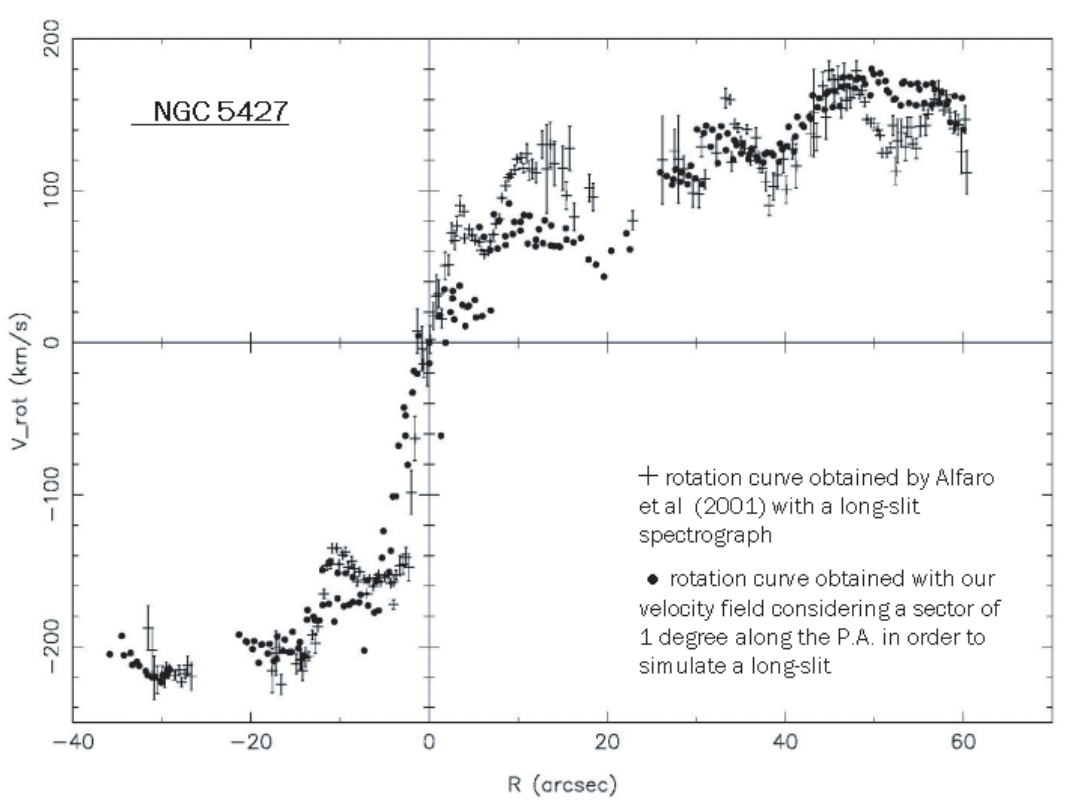

Fig. 8. Comparison between the rotation curve obtained by Alfaro et al. (2001) with a long-slit spectrograph (crosses) and our rotation curve obtained by simulating a long-slit spectrograph rotation curve (dots) considering the same parameters as those authors (PA, $i$, center, angular sector).
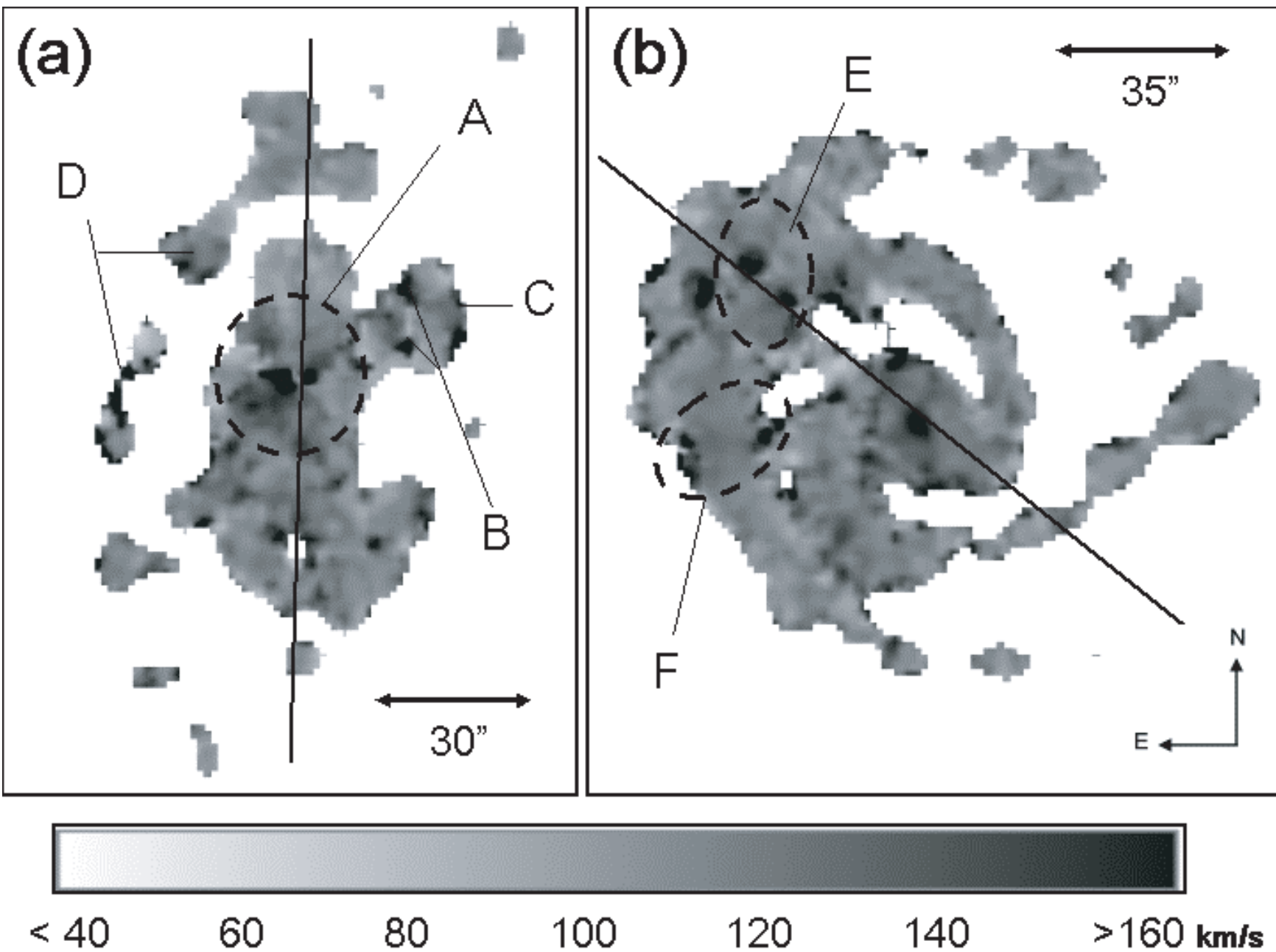

Fig. 9. $F W H M$ fields a) NGC 5426. The black ellipse shows the central cross-like pattern discussed in Sect. 5.1.2. Letters indicate regions with large $F W H M$. b) NGC 5427. Black ellipses display regions discussed in Sect. 5.1.1. Regions E and F show large $F W H M$. 


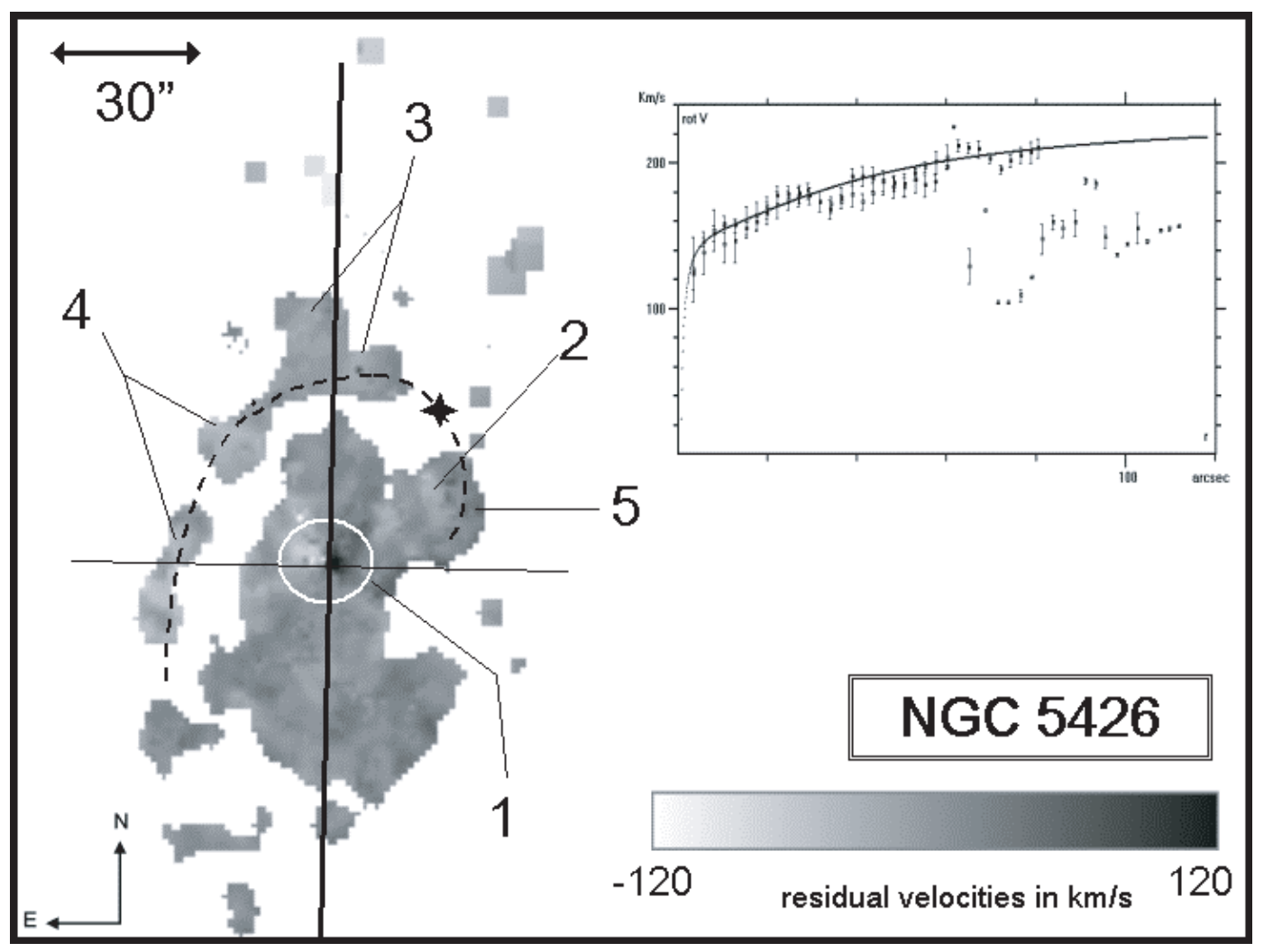

Fig. 10. Residual velocity field of NGC 5426. Upper right panel shows the analytical rotation curve used for the computation of the ideal velocity field. The white circle shows the central part of the galaxy where important residual velocities seem to indicate radial motions of the gas. Regions 1 to 5 are discussed in Sect. 5.2.1. The drawn spiral indicates location of the western spiral arm of the galaxy outlined by stars in Fig. 1a. The black star indicates the point at which large negative residual velocites change their location along the spiral arm (concave to convex) and could be related to the corotation radius of the galaxy.

and such that $\left.V_{\text {rot }}=V_{\text {cir }}+V_{\text {tan }}\right), V_{\text {rad }}$ is the radial velocity component in the plane of the galaxy, and $V_{\perp}$ is the velocity component perpendicular to the plane of the galaxy.

By definition the residual velocity $V_{\text {res }}=V_{\text {ideal }}-V_{\text {obs }}$ at each point is given by

$V_{\text {res }}=-\left[V_{\tan } \times \cos \theta+V_{\text {rad }} \times \sin \theta\right] \times \sin i-V_{\perp} \times \cos i$.

If $V_{\perp} \simeq 0$, the sign of $V_{\text {res }}$ is a combination of the values of $V_{\tan }$, $V_{\text {rad }}$ and $\theta$. For instance along the minor axis, $\theta=90^{\circ}, 270^{\circ} \mathrm{im}-$ plies $V_{\tan }$ contribution is insignificant. For $\theta=90^{\circ}, V_{\text {rad }}$ has the opposite sign of $V_{\text {res }}$, while for $\theta=270^{\circ}, V_{\text {rad }}$ has the same sign as $V_{\text {res }}$. Along the major axis $V_{\text {rad }}$ contribution is insignificant so that for $\theta=180^{\circ}, V_{\tan }$ has the same sign as $V_{\text {res }}$, while for $\theta=0^{\circ}, V_{\tan }$ has the opposite sign of $V_{\text {res }}$.

The analysis of non-circular motions from the residual velocity field is complemented with information from the velocity dispersion field. For instance for low inclinations, $V_{\perp}$ can be neglected in Eq. (2) for regions displaying small velocity dispersion values.

\subsubsection{NGC 5426}

In order to compute the residual velocity field for this galaxy, an analytical curve of the form $V(r) \propto r^{-1} \times\left[r^{1 / 2}+\exp (r)\right]$ was adjusted to the observed rotation curve omitting the points that seemed to be associated with the bridge-like feature. The fit is shown in the upper-right frame in Fig. 10. The ideal velocity field considered was obtained from this rotation curve and Eq. (1). Figure 10 shows the residual velocity field of NGC 5426. This field shows no feature associated with an error in the determination of the kinematical parameters (see discussion in Warner et al. 1973) so that the different velocities displayed in the galaxy correspond to actual non-circular motions of the gas. In the central part (inner $6^{\prime \prime}$ ), there is an important velocity difference. The residual velocities go from $-40 \mathrm{~km} \mathrm{~s}^{-1}$ in the inner $3^{\prime \prime}$ east to the center of the galaxy to $+90 \mathrm{~km} \mathrm{~s}^{-1}$ in the inner 3.5" west of the center (region 1 in Fig. 10). This region matches the incipient bar-like feature detected on the velocity field. An important contribution of negative residual velocities is also seen along the western spiral arm. At the beginning of the spiral arm (region 2 in Fig. 10), this contribution is seen on the concave side of the arm. On the convex side of the same arm, positive residual velocities are found (region 5). Further along the arm, large negative residual velocities are seen on the convex side up to the tip of the arm (regions 3 and 4). This peculiarity could be explained by the acceleration of the gas as it goes through the spiral arm as suggested by Pismis (1986) or to vertical motions also associated to the passage of the gas through the density wave (Alfaro et al. 2001). This contribution of residual velocities could be associated to both types of motion. The point where the position of 


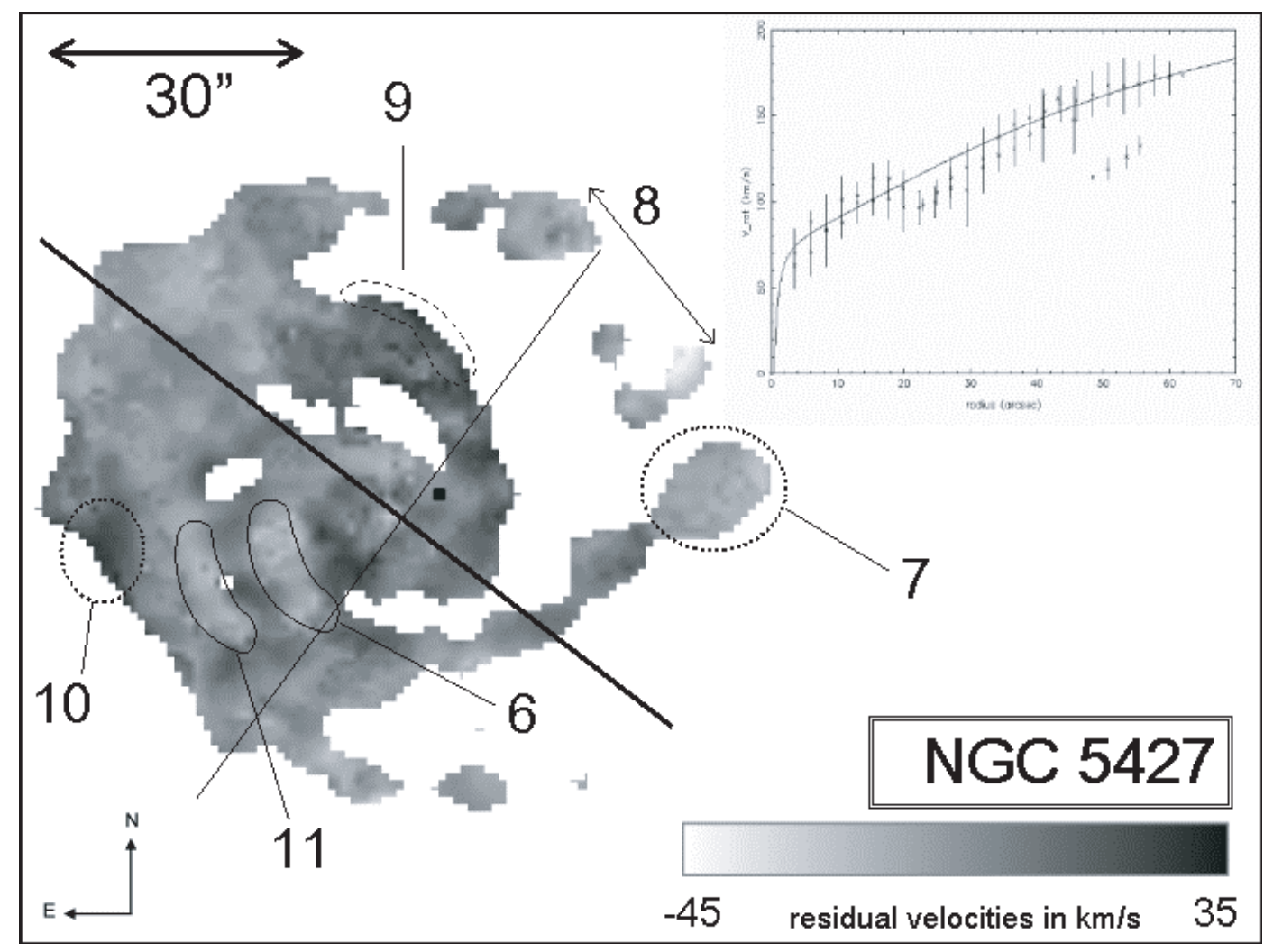

Fig. 11. Residual velocity field of NGC 5427. Upper right panel shows the analytical rotation curve used for the computation of the ideal velocity field. Regions 6 to 11 are discussed in Sect. 5.2.2.

negative velocities changes from concave to convex could be associated to the sense of passage of the gas with respect to the pattern speed of the spiral arms and thus could be associated to the corotation radius of the galaxy (at $R=50^{\prime \prime}$ ). This point is marked with a black star in Fig. 10.

A comparison between the $F W H M$ field and the residual velocity field shows a correlation between $F W H M$ and $V_{\text {res }}$ for the central parts of this galaxy (Figs. 9 and 10) implying an important contribution of non-circular motions. Due to the inclination of the galaxy $\left(i=59^{\circ}\right)$ we cannot explain the large values of the FWHM in the central regions of this galaxy solely by the presence of large perpendicular motions since they could also be the result of important non-circular motions in the plane of the galaxy. Considering that in these regions $\left(\theta \sim 90,270^{\circ}\right)$ there is little contribution of $V_{\tan }$, a fraction of these non-circular motions is probably in the radial direction. Since this region corresponds to the small bar-like feature outlined by the observed velocity field, these non-circular motions could imply gas moving along this structure.

\subsubsection{NGC 5427}

For NGC 5427, the theoretical velocity field was also derived from an analytical curve of the form $V(r) \propto r^{-1} \times\left[r^{1 / 2}+\exp (r)\right]$ fitted to the observed rotation curve omitting the points beyond the bifurcation radius with a low rotation velocity (that is points with $V_{\text {rot }}<115 \mathrm{~km} \mathrm{~s}^{-1}$ beyond $\left.R=46^{\prime \prime}\right)$. The fit is shown in the upper-right frame in Fig. 11.
Regions with $V_{\text {res }}$ different from zero seem to be associated with the spiral arms of the galaxy. Positive residual velocities $V_{\text {res }}>15 \mathrm{~km} \mathrm{~s}^{-1}$ are found in the region between the nucleus of the galaxy and the beginning of the eastern arm (region 6) and the beginning of the western arm (region 11 in Fig. 11). Positive residual velocities larger than $15 \mathrm{~km} \mathrm{~s}^{-1}$ are also seen along the eastern arm of the galaxy (region 8), including the tip of the straight arm segment (region 7). Negative residual velocities are also seen on the outer edges of the western arm (regions 9 and 10). These residual velocities could be associated with the motion of the gas as it encounters, goes through and leaves the density wave as suggested by Pismis (1986). As mentioned by Alfaro et al. (2001), vertical motions should also be present in both cases thus contributing to the non-circular motions at the edges of this spiral arm. None of the regions with large $\left|V_{\text {res }}\right|$ are located along the major or minor axis of the galaxies, so no further analysis can be done using Eq. (3).

\subsection{Kinematical vs. morphological features}

\subsubsection{NGC 5426}

The bridge-like feature between both galaxies was analyzed by comparing the location in the projected plane of each emitting region with its associated radial velocity. Only regions $\mathrm{M}$ and $\mathrm{N}$ (Fig. 4) display radial velocities closer to those of NGC 5427 and could actually belong to this galaxy's disk. Other regions that seem to be closer to NGC 5427 than to NGC 5426 - in projection - display velocities closer to those of NGC 5426 
(regions $\mathrm{G}$ to $\mathrm{L}$ ). We analyzed the influence of each of these regions on NGC 5426's rotation curve (Figs. 4 and 5) in an attempt to tell which of these regions still follow the main motion of the galaxy's rotation and which regions seem to be suffering the effects of tidal forces due to the interaction. Regions A and $\mathrm{B}$ still follow the global behavior of the rotation curve, region $\mathrm{F}$ shows a decrease of $20 \mathrm{~km} \mathrm{~s}^{-1}$ in the rotation velocity while adjacent regions $\mathrm{D}, \mathrm{E}, \mathrm{G}$ and $\mathrm{J}$ are clearly below the average rotation velocity of the galaxy $\left(V_{\text {rot }} \sim 110 \mathrm{~km} \mathrm{~s}^{-1}\right)$ compared to an average value of $\sim 200 \mathrm{~km} \mathrm{~s}^{-1}$ in parts of the galaxy which seem to display a circular motion. At larger radii, regions $\mathrm{C} 1, \mathrm{C} 2, \mathrm{I}, \mathrm{K}$ and $\mathrm{L}$ show a slight increase of $\sim 30 \mathrm{~km} \mathrm{~s}^{-1}$ but they still remain below the average rotation velocity. Only region $\mathrm{H}$ shows a velocity value closer to the average. In the projected plane, regions A and B do seem closer to NGC 5426 and could actually belong to this galaxy's disk. Nevertheless regions E, D, G and J seem to be closer to NGC 5426 than regions $\mathrm{K}$ and $\mathrm{L}$ whose velocities are closer to those of the average rotation curve. This could give a clue as to the actual position of the bridge-like feature in a 3D scenario. Regarding the inner structure of the galaxy, kinematical information from the rotation curve is not available for the inner $7 "$ of this galaxy, so any eventual effect of the small bar-like feature $\left(\sim 3.0^{\prime \prime}\right.$ in semi-length) on the rotation curve is not visible.

\subsubsection{NGC 5427}

For the approaching side of this galaxy, most of the oscillations observed can be associated to the HII regions along the arms of the galaxy (Figs. 7 and 12). For instance, the small bump around a radius of $16^{\prime \prime}$ corresponds to the beginning of the western arm and is marked by a small HII region (region $U$ in Fig. 12). This radius matches the semi-length of the central bar. The velocity then decreases by $\sim 10 \mathrm{~km} \mathrm{~s}^{-1}$ which corresponds to the interarm region marked as $X$. After this region, the rotational velocity begins to increase rather steadily once we reach the straight segment of the eastern arm of the galaxy (region between $\mathrm{V}$ and $\mathrm{W}$ ). We then notice an abrupt decrease in the velocity of about $40 \mathrm{~km} \mathrm{~s}^{-1}$ (region Y) between the second-to-last HII region (W) and the last HII region of the straight spiral arm segment (region Z) as well as the tip of the western arm (regions N). The tip of this arm is marked by a very intense and large HII region where important star formation is going on (region Z). This side of NGC 5427 is closer to the companion, so this decrease of the rotational velocity could be due to the slowing-down and homogenization of velocities along the straight arm and/or to the fact that this part of the arm no longer lies in the main plane of the galaxy-both these processes being caused by the interaction. On the receding side of the rotation curve, the end of the bar also matches a small bump feature (region $\mathrm{T}$ ). The rotational velocity decreases at the interarm region $(\mathrm{O})$. Following this decrease, the velocity increases forming a slight hump from the bifurcation of the western arm (P) - marked by an HII region - to larger and more intense HII regions along this arm (regions $\mathrm{R}$ and $\mathrm{S}$ ). This hump then reaches a slight plateau once the outermost arm segment of the western arm (Q) is reached. At $R=16^{\prime \prime}$ on both

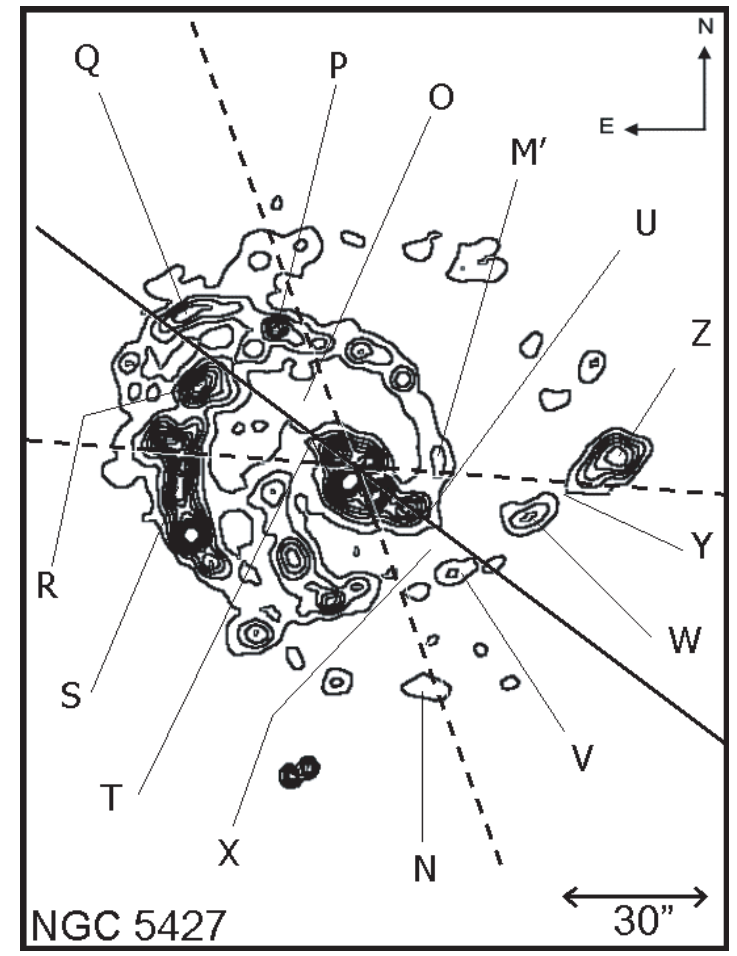

Fig. 12. Monochromatic isophotes of NGC 5427. Letters indicate features associated to variations in this galaxy's rotation curve shown in Fig. 7. Solid line indicates the galaxy's position angle (PA), the slashdotted lines indicate the angular sector from both sides of the major axis considered for the computation of the galaxy's rotation curve.

sides of the rotation curve, the end of the bar is marked by a small plateau of $\sim 110 \mathrm{~km} \mathrm{~s}^{-1}$ for the receding side (region T) and of $\sim 100 \mathrm{~km} \mathrm{~s}^{-1}$ for the approaching one (region $\mathrm{U}$ ).

\section{Dynamical analysis}

\subsection{Mass estimates}

For each galaxy, a range of possible masses was computed using the method proposed by Lequeux (1983). This method considers two extreme cases to evaluate a galaxy's mass: the galaxy viewed as a flat disk and the galaxy viewed as a spherical system. For the flat disk case, the formulas from the mass model by Nordsieck (1973) and a theoretical rotation curve that stays flat after the solid-body rotation section are used as assumptions to approximate the mass inside a radius $R$ by $M(R)=0.6 \times\left(R V^{2}(R) / G\right)$. On the other side for any spherical model, the mass inside a certain radius is always given by $M(R)=R V^{2}(R) / G$. For a real disk galaxy, the actual value of $M(R)$ should always lie between these two extreme values. In fact, if a massive halo is considered, the expression considered for the spherical model should be the most appropriate for the determination of a galaxy's mass.

The overall rotation curves of these galaxies cannot be considered as flat. Nevertheless since this is an early interaction, circular motions of the gas dominate the inner parts of the galaxy so that up to a certain radius the rotation curve for both sides of the galaxy is representative of circular motion in which case Lequeux's method may be applied. In order to determine 
Table 3. Mass estimates for NGC 5426 and NGC 5427.

\begin{tabular}{|c|c|c|c|c|c|c|c|}
\hline & $\begin{array}{c}R_{\mathrm{bif}}^{a} \\
\text { in } \mathrm{kpc}\end{array}$ & $\begin{array}{c}V\left(R_{\mathrm{bif}}\right) \\
\text { in } \mathrm{km} \mathrm{s}^{-1}\end{array}$ & $M\left(R_{\text {bif }}\right) \times 10^{10} M_{\odot}$ & - & $\begin{array}{l}D_{25} / 2 \\
\text { in } \mathrm{kpc}\end{array}$ & $\begin{array}{l}V\left(D_{25} / 2\right) \\
\text { in } \mathrm{km} \mathrm{s}^{-1}\end{array}$ & $M\left(D_{25} / 2\right) \times 10^{10} M_{\odot}$ \\
\hline NGC 5426 & 7.8 & 198 & $4.05-6.75$ & - & 11.5 & 208 & $6.72-11.2$ \\
\hline NGC 5427 & 6.0 & 154 & $1.92-3.20$ & - & 11.1 & 172 & $4.5-7.5$ \\
\hline
\end{tabular}

${ }^{a}$ Radius at which symmetry on the rotation curve (approaching vs. receding side) is lost.

this radius, we looked for the radius on the rotation curve, $R_{\mathrm{bif}}$ at which symmetry of the approaching and the receding sides is dramatically lost $\left(\left|V_{\text {receding }}-V_{\text {approaching }}\right| \geq 40 \mathrm{~km} \mathrm{~s}^{-1}\right)$.

For NGC 5426 (Fig. 5), symmetry is lost after $R_{\text {bif }}=60^{\prime \prime}$, the velocity at this radius is $198 \mathrm{~km} \mathrm{~s}^{-1}$. Considering the distance modulus given by de Vaucouleurs (1979) for NGC 5427 is also valid for this galaxy, then $R_{\text {bif }}=7.8 \mathrm{kpc}$ so that up to this radius this galaxy's mass lies between $4.05 \times 10^{10} M_{\odot}$ and $6.75 \times 10^{10} M_{\odot}$. For NGC 5427 (Fig. 7), $R_{\text {bif }}=46.5^{\prime \prime}$ with $V=154 \mathrm{~km} \mathrm{~s}^{-1}$. Considering the same distance modulus, $R_{\text {bif }}=6.02 \mathrm{kpc}$, so the mass up this radius lies between $1.92 \times 10^{10} M_{\odot}$ and $3.20 \times 10^{10} M_{\odot}$. Results are shown in Table 3.

Furthermore, although for both rotation curves the side of the curve closest to the companion shows dramatic variations after $R_{\text {bif }}$, the other side of each curve shows no distinct difference before and after this bifurcation radius is reached. For both galaxies, the rotation curve seems to flatten down at large radii after reaching a maximum velocity $V_{\max }$ (Figs. 5 and 7). One may then assume that for each of these sides, the rotation curve is representative of circular motion up to the last detected emission point and that it can be extrapolated up to $D_{25} / 2$ by assigning to this radius the maximum rotational velocity observed $V_{\max }$. For NGC 5426, $V_{\max }=208 \mathrm{~km} \mathrm{~s}^{-1}$ and $D_{25} / 2=$ $88.6^{\prime \prime}=11.47 \mathrm{kpc}$, for NGC 5427, $V_{\max }=172 \mathrm{~km} \mathrm{~s}^{-1}$ and $D_{25} / 2=85.5^{\prime \prime}=11.06 \mathrm{kpc}-$ both $D_{25} / 2$ values were taken from the LEDA database. The resulting individual masses computed with these values are $M_{\mathrm{NGC}} 5426=1.12 \times 10^{11} M_{\odot}$ and $M_{\text {NGC } 5427}=0.75 \times 10^{11} M_{\odot}$ Results are summarized in Table 3 .

The combined mass of the components from the orbital motion of the pair was also computed using the expression given by Karachentsev \& Mineva (1984) for a statistical estimate of the orbital mass:

$M_{\text {orbital }}=\frac{32}{3 \pi}\left(\frac{\Delta V^{2} \times X_{12}}{G}\right)$

where $\Delta V$ is the difference between the systemic velocities of the galaxies, $X_{12}$ is the projected separation from one nucleus to the other, $G$ is the gravitational constant and $32 / 3 \pi$ is the mean value of the projection factor for circular motions of the members of the pair and isotropic orientation of the orbits. For NGC 5426 and NGC 5427, we have $\Delta V=147.5 \mathrm{~km} \mathrm{~s}^{-1}$ and $X_{12}=18.17 \mathrm{kpc}$, so the orbital mass equals $3.01 \times 10^{11} M_{\odot}$.

Comparing the orbital mass with the sum of the individual masses we find that $M_{\text {add }}=M_{\mathrm{NGC} 5426}+M_{\mathrm{NGC} 5427}=1.87 \times$ $10^{11} M_{\odot}$, so that $M_{\text {add }} / M_{\text {orbital }}=0.62$. It should be kept in mind though that Eq. (4) is a statistical estimate of the orbital mass of the pair under certain assumptions and such that the lower mass limit for a circular orbit is about $1 / 3$ of this value and for a parabolic orbit the value is even smaller.

\subsection{Mass distribution}

The mass model used in this work was taken from Blais-Ouellette et al. (2001). It considers both the light distribution of the stars and a theoretical dark halo profile to compute a rotation curve that best fits the observed one. The density of the theoretical dark matter halo is given by the following expression (Blais-Ouellette et al. 2001):

$\rho(R)=\frac{\rho_{0}}{\left[c+\left(R / R_{0}\right)^{\gamma}\right]\left[1+\left(R / R_{0}\right)^{\alpha}\right]^{(\beta-\gamma) / \alpha}}$

where $\rho_{0}$ is a characteristic density, $R_{0}$ is a characteristic radius and $c$ can force the presence of a flat-density core. The parameters $\alpha, \beta$ and $\gamma$ determine the shape of the density profile. For a pseudo-isothermal sphere (Begeman 1987), $c=1, \alpha=1$, $\beta=2$ and $\gamma=2$; for a NFW profile (Navarro et al. 1996), $c=0, \alpha=1, \beta=3$ and $\gamma=1$ and for an isothermal sphere (Kravtsov et al. 1998), $c=0, \alpha=2, \beta=3$ and $\gamma=0$. In this way, the $M / L$ as well as the dark matter halo properties: central density $\rho_{0}$ and core radius $R_{0}$, are free parameters. For both galaxies optical photometry in the $B$ band was taken from Wozniak et al. (1995).

For NGC 5426, none of the models considered (maximum disk, submaximum disk or best fit model; pseudoisothermal, NFW or isothermal dark halo profile) fits the three inner points of the observed rotation curve. Figure 13 shows the fit with the lowest computed $\chi^{2}$, it considers a maximum disk $(M / L=11.15)$ and a pseudo-isothermal sphere for the dark halo. The inner points of the rotation curve could only be fitted by assuming the contribution of the disk is almost negligeable $(M / L=0.1)$ and considering a pseudo-isothermal halo with a large central density, $\rho_{0}=0.005 M_{\odot} \mathrm{pc}^{-3}$. One possible, disk-related, reason why the inner rotation curve of NGC 5426 cannot be fitted is that the disk contribution considered for this fit was taken from a $B$-image while the central regions of the galaxy might have larger $M / L$ than the rest of the disk (the bulge region is probably "redder").

For NGC 5427, the best fit model gives $(M / L)_{B}=1.7$ (maximal disk) - which matches the value found by Blackman (1982) - and an isothermal halo implying an important dark matter contribution in the outer parts of this galaxy. The divergence between the data and the model correspond to regions associated to the spiral arms (Fig. 14).

The previously computed ratio $M_{\text {add }} / M_{\text {orbital }}$ of 0.62 is consistent with an important contribution of unseen matter in 


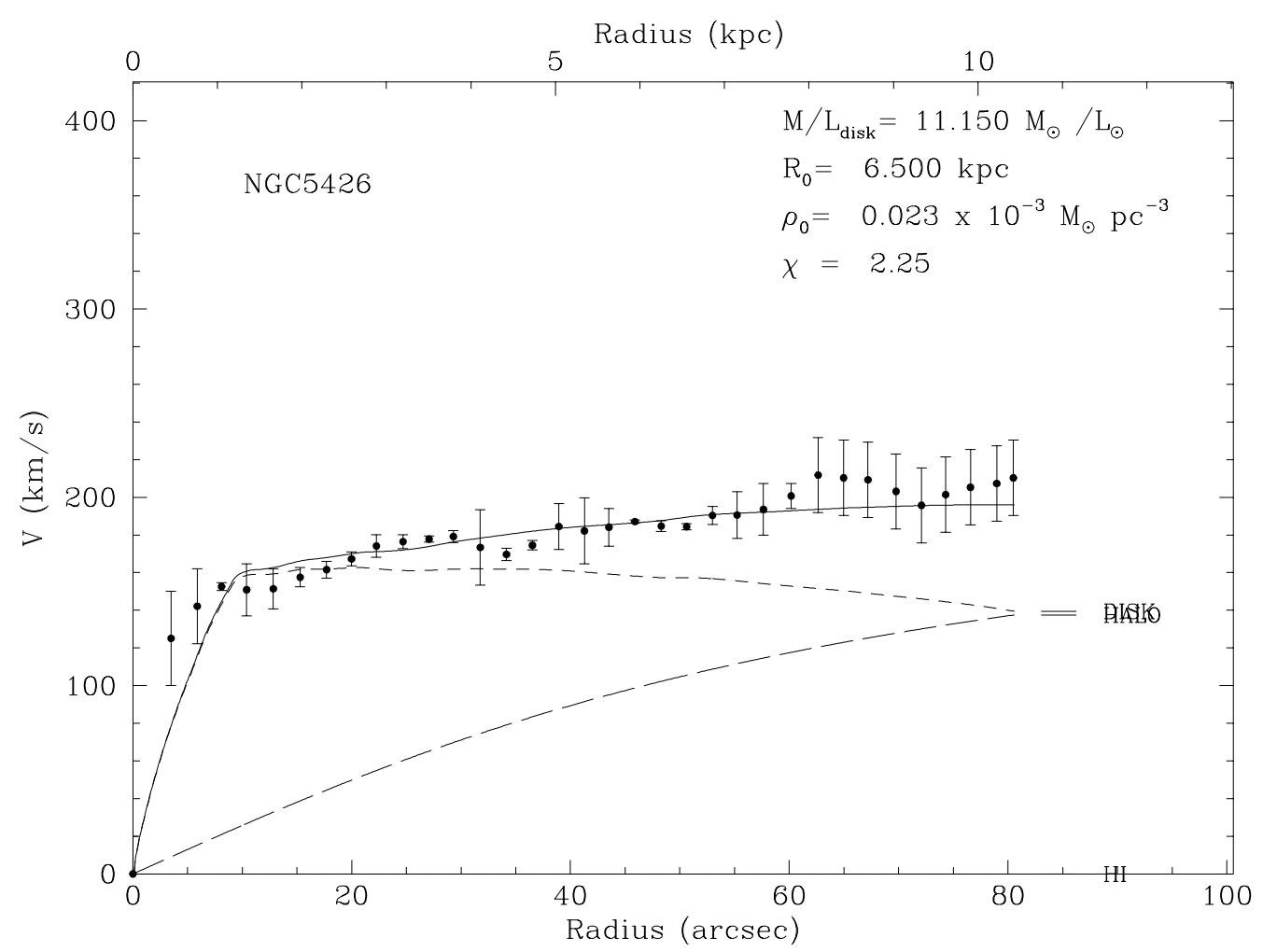

Fig. 13. Best mass model fit for NGC 5426 using a pseudoisothermal halo and a maximum disk. Long-dashed curve represents the dark-matter halo contribution, short-dashed curve represents the stellar disk contribution. The parameters displayed in each curve stand for the mass-to-light ration of the stellar disk $\left(M / L_{\text {disk }}\right)$, the core radius of the dark matter halo and its central density $\left(R_{0}\right.$ and $\rho_{0}$, respectively) and the minimized $\chi^{2}$ in the three-dimensional parameter space. Mass-model taken from Blais-Ouellette et al. (2001).

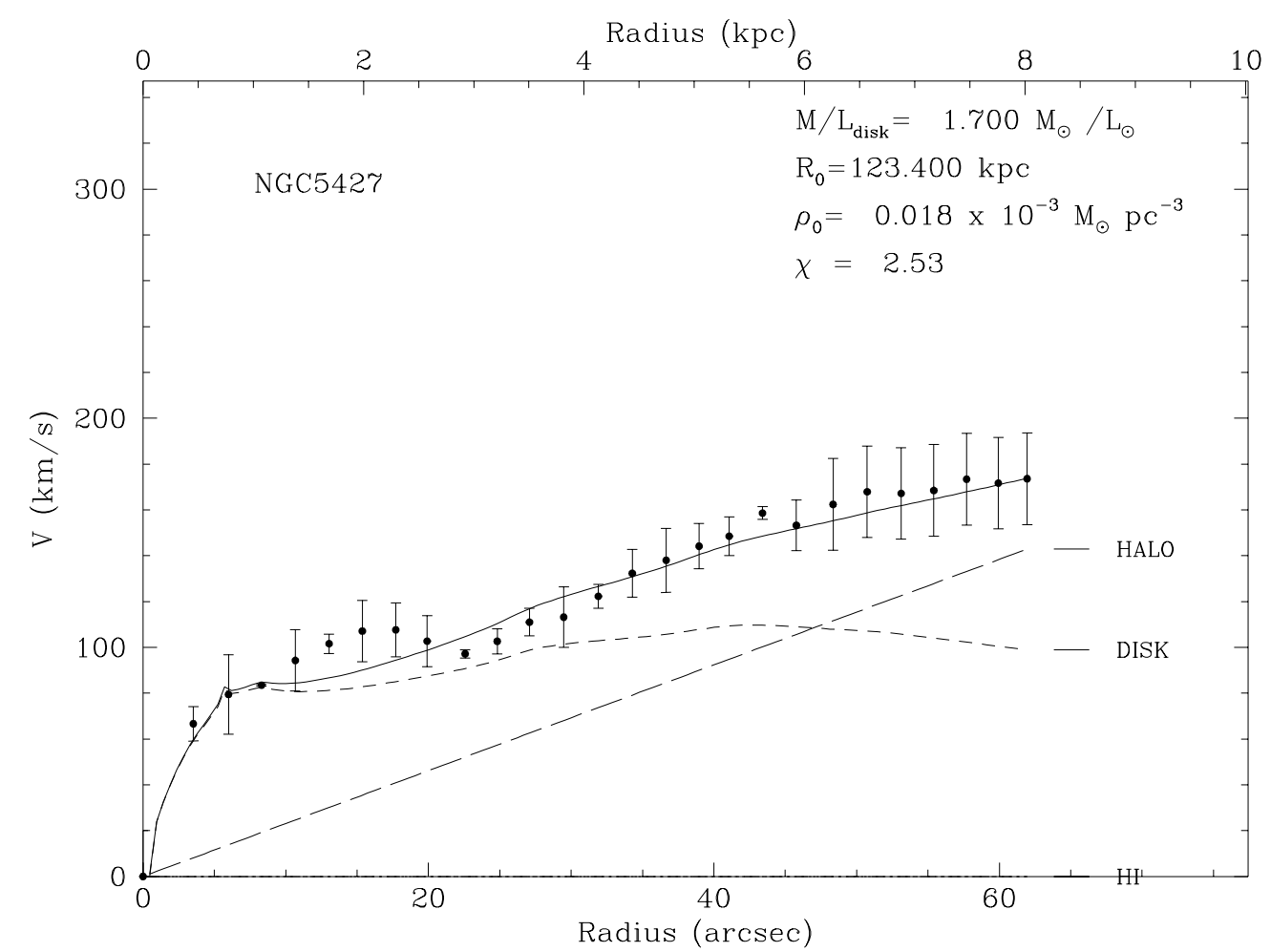

Fig. 14. Maximum disk mass model for NGC 5427 using an isothermal halo. The parameters displayed in each curve stand for the mass-to-light ration of the stellar disk $\left(M / L_{\text {disk }}\right)$, the core radius of the dark matter halo and its central density $\left(R_{0}\right.$ and $\rho_{0}$, respectively) and the minimized $\chi^{2}$ in the three-dimensional parameter space. Mass-model taken from Blais-Ouellette et al. (2001). 
the outermost parts of the galaxies that cannot be acquainted through the computation of each galaxy's mass using the optical $\mathrm{H} \alpha$ rotation curve and Lequeux's method. On the other hand the mass of NGC 5427 might have been uderestimated due to an overestimation of its inclination. The uncertainty in inclination determination has a big effect on the derived velocity amplitude, and thus masses and $M / L$ ratios: for example, $1 / \sin 34^{\circ}=1.79$ and $1 / \sin 24^{\circ}=2.46$, indicating already a factor of 1.9 difference in masses. This would explain why even if within smaller radii NGC 5426 seems to be more massive, the radial velocities of the bridge-like feature between both galaxies seems to belong to the latter implying it is more affected by the interaction than the companion NGC 5427. The fact that probably these two galaxies have approximately the same masses supports the idea of them being twin galaxies as suggested by Yamagata et al. (1989).

\section{Discussion}

As suggested by Blackman (1982), the asymmetric distribution of light of the pair could be due to the fact that NGC 5427 has not had enough time to average the distribution of its outer parts. Considering that for this galaxy $V_{\text {rot }} \sim 172 \mathrm{~km} \mathrm{~s}^{-1}$ at $R \sim 9 \mathrm{kpc}$, the rotation period would amount to $\sim 3 \times 10^{8}$ yrs thus sustaining Blackman (1982)'s suggestion of this being a "recent" encounter. Nevertheless Schombert et al. (1990) showed that the bridges and tails of interacting galaxies are bluer than the inner disks showing that on average star formation has increased in the interaction zone. Also, $N$-body models of interacting galaxies show star formation is induced simultaneously in all azimuthal directions. These two last results (found after Blackman's work) do not support the idea that the surface brightness in the interaction zone between the two galaxies is weaker than on the opposite sides of the galaxies, because the pair has not had time to average the star formation in these galaxies. A second and more convincing explanation for the lack of emission on the adjacent halves of the galaxies was also given by Blackman (1982). According to him, this lack of emission could be explained by obscuration of dust in NGC 5426's disk whose inclination is larger than that of NGC 5427's. This would imply NGC 5426 is in front of NGC 5427.

NGC 5426 is a two-armed spiral galaxy with well-defined arms so we may assume it is a trailing spiral since leading spiral arms are an uncommon phenomena (Pasha \& Smirnov 1982) and the dynamical theory to explain them is mostly associated to the formation of a single leading arm (Thomasson et al. 1989). Considering this sens of rotation and the position of the approaching and the receding radial velocities we can infer this galaxy's orientation placing its western side closer to the observer than the eastern side. For NGC 5427 we can use the same argument to determine it is a trailing spiral. This spin is also suggested by Alfaro et al. (2001) according to the movement of the gas as it goes through the density wave. Considering the distribution of approaching and receding radial velocities, the southeastern side of the galaxy is closer to the observer than the northwestern one. From both the tilt and spin of the galaxies as well as from the position of one with respect to the other a possible sense of passage of the encounter can be suggested. The fact that NGC 5427 is a two-arm grand-design spiral suggests the encounter with NGC 5426 is a direct one. Comparing the morphology of this pair with the simulations of Toomre \& Toomre (1972) we find that the bridge-like feature observed in Arp 271 resembles the elongation presented by these authors in the -0.5 frame of their Fig. 3 which corresponds to the first stages $\left(0.5 \times 10^{8}\right.$ yrs before perigalactic instant $)$ of a flat direct parabolic passage of a companion of equal mass. Though this is a loose comparison and the system is likely to be more complicated, we think this first analysis can help as a starting point for future numerical simulations of this particular encounter. The suggested global geometry of the encounter as well as the sense of passage are shown in Fig. 15.

As already done by Blackman (1982), the time scale of the interaction was estimated from the ratio of the projected separation between both galaxies $(18.17 \mathrm{kpc})$ and the difference in the systemic velocities of the pair $\left(147.5 \mathrm{~km} \mathrm{~s}^{-1}\right)$ resulting in $\sim 10^{8} \mathrm{yrs}$, a value which is also similar to the time-scale in the Toomre \& Toomre (1972) simulation. This result implies the interaction is not necessarily very strong, in agreement with the fact that the galaxies are not very distorted. This makes it possible to assume that the noncircular motions are not necessarily very strong so that it is possible thus validating the mass estimates for the galaxies. However, there do exist characteristics that can be associated to galaxy interaction process such as the bridge of material, the distorted velocity field in NGC 5427 and its straight spiral arm segment. The range of radial velocities observed along the straight arm is considerably smaller than in the other parts of this galaxy's arms as if the whole structure were slowing down to an average velocity due to the proximity with NGC 5426 and/or as if this part of the spiral structure is being deviated from the galactic plane during the interaction. The small shift between the central isovelocity contours in NGC 5426 seems to be outlining a small and incipient central bar of about 6 " whose formation could also have been triggered by the interacting process. The giant HII regions in each galaxy could also be sign of recent star formation triggered by the interacting process. Nevertheless, these peculiarities could also be due to the intrinsic nature of spiral galaxies. For example, the origin of bars in interacting disk galaxies remains unclear. Though there have been works showing statistical evidence that companions trigger the formation of bars (Elmegreen et al. 1990; Marquez et al. 2000), bars can be formed as a result of disk instabilities regardless of their environment (van den Bergh 2002). For example, it was shown by Hohl (1971) that cool stellar disks are very sensitive to bar formation, the so-called "bar instability" scenario later decribed by Sellwood \& Wilkinson (1993). On the other hand, Noguchi (1987) and Miwa \& Noguchi (1998) have shown by $N$-body simulations that galaxy interaction can trigger bar formation. Detailed numerical simulations considering strict kinematical restrictions need to be done to establish the extent to which the interacting process is related to the formation of a possible nuclear bar - both in the case of NGC 5427's central bar and of NGC 5426's small incipient bar-like feature.

Another interesting feature observed in Arp 271 is the fact that the bridge of stars does not appear to match or follow 


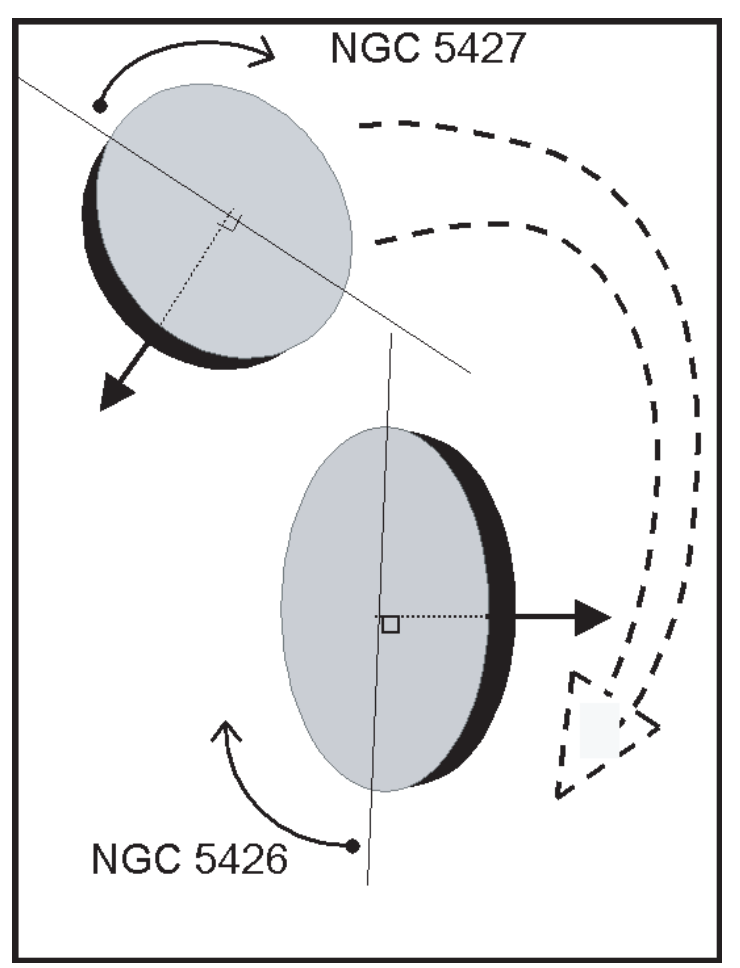

Fig. 15. Possible 3D configuration for the encounter between NGC 5426 and NGC 5427. The spin and orientation of each galaxy is indicated. The large arrow indicates the possible sense of passage of one galaxy with respect to the other where NGC 5427 is moving from behind NGC 5426 towards the foreground in the bottom-right corner of the frame.

any other structure in neither two galaxies. In fact, this bridge is almost perpendicular to the linear segment in the southern arm of NGC 5427 and it does not quite seem to follow the western arm of NGC 5426. This peculiarity has been observed in other galaxies where tidal tails, bridges and spiral structure coexist, yet do not seem to join smoothly. In systems such as Arp 96 and M100, the disk's spiral structure appears to have begun to decouple from the tidal structure (Schweizer 1998). These differences between gas and stars could be due to non-gravitational forces such as ram-pressure due to hot gas in quiescent halos, starburst-driven outflows or magnetic fields. In our case, this could also be some part of the spiral structure deviated from the galactic plane during the interaction. The study of galaxies showing this peculiarity can give us information about the kinematical and dynamical processes that lead to the formation and sustainment of grand design spiral structure and the parameters that control it. The fact that the systemic velocities differ by almost $150 \mathrm{~km} \mathrm{~s}^{-1}$ and that the galaxies seem to be connected by a bridge of material suggests that we are actually measuring an important component of the peculiar velocities between these two galaxies. This relative radial velocity difference might represent a significant component of the total velocity difference. So, if we assume for a moment that the skyplane velocity components are exactly zero, NGC 5426 would "see" the other galaxy passing away from observer, thus in an almost retrograde sense with respect to its internal rotation (orbital inclination about 150 degrees), and similarly NGC 5427 would see an almost perpendicular passage of the other galaxy. The almost perpendicular passage seen by NGC 5427 would be consistent with the absence of apparent tails or bridges emanating from it - but might still help to excite spiral structure. The almost retrograde passage seen by NGC 5426 might explain the weakness of bridges.

\section{Conclusions}

The detailed study of the kinematics and dynamics of interacting galaxy pairs provides important information about the structure of each member of the pair as well as of the encounter as a whole. In this work we presented Fabry-Perot observations of the isolated interacting galaxy pair NGC 5426/27 (Arp 271) showing that for a perturbed system it is important to have kinematical information of large portions of the galaxies studied. From the analysis of the velocity field and the computation of the $F W H M$ of the intensity profiles and residual velocities maps, we determined non-circular motions in each galaxy and found them to be possibly associated with the passage of gas through the spiral arms and also with the flow of gas into or out of the central parts of each galaxy along bar-like features. From the observed rotation curve of each galaxy and the fit of different mass models, we found that though NGC 5426 seems to be more massive than NGC 5427 within its optical radius, both galaxies seem to have approximately the same total mass, thus belonging to the twin galaxies scheme. For NGC 5427 the observed rotation curve can be fitted with a maximum disk $(M / L=1.7)$ and a pseudo-isothermal halo. Though this seems to be a relatively early encounter, several morphological features of each galaxy were associated with the interaction process, such as an incipient bar-like feature in the central parts of NGC 5426 and a straight segment in one of NGC 5427's arms. The radial velocities of the HII regions outlining a bridge between the two galaxies were also derived finding that this structure seems to be associated with NGC 5426. Finally from the resulting kinematics and dynamics, we suggest a possible 3D configuration for this encounter. This will be used as a starting point in future numerical simulations of an encounter of this sort.

Acknowledgements. We thank the staff of the OAN (Observatorio Astronómico Nacional) for their support during PUMA data acquisition. We acknowledge the financial support from grant IN 104696 of DGAPA-UNAM. We also acknowledge C. Carignan for allowing us to use his mass model. I. Fuentes-Carrera acknowledges financial support from Fundación UNAM and CONACYT and DGEP grants. I. Fuentes.Carrera also thanks the Observatoire de Marseille for its hospitality during several research stays with financial support from ECOS(France)/ANUIES(Mexico).

\section{References}

Alfaro, E. J., Pérez, E., González-Delgado, R. M., Martos, M. A., \& Franco, J. 2001, ApJ, 550, 253

Amram, P., Marcelin, M., Balkowski, C., et al. 1994, A\&AS, 103, 5 Amram, P., Balkowski, C., Boulesteix, J., et al. 1996, A\&A, 310, 737 Amram, P., Mendes de Oliveira, C., Plana, H., et al. 2002, A\&AS, 281, 389 
Arp, H. 1966, Atlas of Peculiar Galaxies (Pasadena: California Institute of Technology)

Barnes, J. E. 1988, ApJ, 331, 699

Barnes, J. E., \& Hernquist, L. 1996, ApJ, 471, 115

Begeman, K. G. 1987, Ph.D. Thesis, Gronigen University

Blackman, C. P. 1982, MNRAS, 200, 407

Blais-Ouellette, S. 2000, Ph.D. Thesis, Université de Montreal, Université de Provence

Blais-Ouellette, S., Amram, P., \& Carignan, C. 2001, AJ, 121, 1952

Bottinelli, L., Gouguenheim, L., Paturel, G., \& De Vaucouleurs, G. 1984, A\&AS, 56, 381

Chengalur, J. N., Salpeter, E. E., \& Terzian, Y. 1995, AJ, 110, 167

de Vaucouleurs, G. 1979, ApJ, 227, 729

de Vaucouleurs, G., de Vaucouleurs, A., Corwin, J. R., et al. 1991, Third Reference Catalogue of Bright Galaxies (New York: Springer-Verlag)

Elmegreen, D. M., Elmegreen, B. G., \& Bellin, A. D. 1990, ApJ, 364, 415

González-Delgado, R. M., \& Pérez, E. 1992, Relationships Between Active Galactic Nuclei and Starburst Galaxies, ed. V. Filippenko, PASP, 31, 371

Hohl, F. 1971, ApJ, 168, 343

Honma, M. 1999, ApJ, 516, 693

Hubble, E. 1943, ApJ, 97, 112

Jungwiert, B., Combes, F., \& Axon, D. J. 1997, A\&AS, 125, 479

Karachentsev, I. D. 1972, Catalogue of isolated pairs of galaxies in the northern hemisphere, Soobshch. Spets. Astrofiz. Obs., 7, 1

Karachentsev, I. D., \& Mineva, V. A. 1984, Sov. Astron. Lett., 10, 4

Kaufman, M., Brinks, E., Elmegreen, B. G., et al. 1999, AJ, 118, 1577

Keel, W. C. 1996, AJ, 111, 696

Kravtsov, A. V., Klypin, A. A., Bullock, J. S., \& Primack, J. R. 1998, ApJ, 502, 48

Le Coarer, E., Rosado, M., Georgelin, Y., Viale, A., \& Goldes, G. 1993, A\&A, 280, 365

Lequeux, J. 1983, A\&A, 125, 394

Lin, C. C., \& Shu, F. H. 1964, ApJ, 140, 646

Marcelin, M., Le Coarer, E., Boulesteix, J., Georgelin, Y., \& Monnet, G. 1987, A\&A, 179, 101

Marquez, I., Durret, F., Masegosa, J., et al. 2000, A\&A, 360, 431
Miwa, T., \& Noguchi, M. 1998, ApJ, 499, 149

Navarro, J. F., Frenk, C. S., \& White, S. D. M. 1996, ApJ, 462, 563

Noguchi, M. 1987, MNRAS, 228, 635

Nordgren, T. E., Chengalur, J. N., Salpeter, E. E., \& Terzian, Y. 1998, ApJS, 115, 43

Nordsieck, K. H. 1973, ApJ, 184, 719

Page, T. 1975, in Stars and Stellar Systems vol. 9, Galaxies and the Universe, ed. A. Sandage, M. Sandage, \& J. Kristian (Chicago: The University of Chicago Press), 541

Pasha, I. I., \& Smirnov, M. A. 1982, Ap\&SS, 86, 215

Pismis, P. 1986, RMxAA, 12, 79

Rosado, M., Langarica, R., Bernal, A., et al. 1995, RMxAA (Serie de Conferencias), 03, 263

Salo, H., \& Laurikainen, E. 1993, ApJ, 410, 586

Salo, H., \& Laurikainen, E. 2000, MNRAS, 319, 377

Salo, H., \& Laurikainen, E. 2000, MNRAS, 319, 393

Sandage, A., \& Bedke, J. 1994, The Carnegie Atlas of Galaxies, vol. II, Carnegie Institution of Washington with The Flintridge Foundation

Schombert, J. M., Wallin, J. F., \& Struck-Marcell, C. 1990, AJ, 99, 497

Schweizer, F. 1998, in Galaxies: Interactions and Induced Star Formation, Saas-Fee Advanced Course 26, ed. R. C. Kennicutt, Jr. F. Schweizer, et al., 105

Schweizer, L. Y. 1987a, ApJS, 64, 411

Schweizer, L. Y. 1987b, ApJS, 64, 427

Sellwood, J. A., \& Wilkinson, A. 1993, Rep. Prog. Phys., 56, 173

Thomasson, M., Donner, K. J., Sundelius, B., et al. 1989, A\&A, 211, 25

Toomre, A., \& Toomre, J. 1972, ApJ, 178, 623

van den Bergh, S. 2002, AJ, 124, 782

Vorontsov-Velyaminov, B. A. 1959, Atlas and Catalogue of Interacting Galaxies, part I (Moscow: University of Moscow)

Warner, P. J., Wright, M. C. H., \& Baldwin, J. E. 1973, MNRAS, 163, 163

Wozniak, H., Friedli, D., Martinet, L., Martin, P., \& Bratschi, P. 1995, A\&AS, 111, 115

Yamagata, T., Noguchi, M., \& Iye, M. 1989, ApJ, 338, 707 


\section{Online Material}


I. Fuentes-Carrera et al.: Interacting galaxy pair Arp 271, Online Material p 2

Table 1. Instrumental and observational parameters.

\begin{tabular}{ll}
\hline \hline Parameter & Value \\
\hline Telescope & $2.1 \mathrm{~m}($ OAN, S PM $)$ \\
Instrument & PUMA \\
Detector & Tektronic CCD \\
Detector size & $(1024 \times 1024) \mathrm{px}$ \\
Image scale (after $2 \times 2$ binning $)$ & $1.18{ }^{\prime \prime}$ per px \\
Scanning F-P interferometer & ET $-50($ Queensgate $)$ \\
F-P interference order at H $\alpha$ & 330 \\
Free spectral range at H $\alpha$ & $19.95 \AA\left(912 \mathrm{~km} \mathrm{~s}^{-1}\right)$ \\
Spectral sampling at H $\alpha$ & $0.41 \AA\left(19.0 \mathrm{~km} \mathrm{~s}^{-1}\right)$ \\
Interference filter & $6650 \AA($ FWH $=47 \AA)$ \\
Total exposure time & $96 \mathrm{~min}$ \\
Exposure time (direct image) & $120 \mathrm{~s}$ \\
Calibration line & $6678.15 \AA(\mathrm{He}$ lamp) \\
\hline
\end{tabular}

